International Journal of Applied Mathematical Research, 1 (1) (2012) 77-107

CScience Publishing Corporation

www.sciencepubco.com/index.php/IJAMR

\title{
Piecewise Analytic Method
}

Tamer A. Abassy

\author{
Department of Scientific Computing,Faculty of Computers and Informatics, \\ Benha University, Benha 13518, Egypt. \\ Department of mathematics,Faculty of Arts and Science in Wadi Addwasir, \\ Salman Bin Abdulaziz University, KSA. \\ E-mail: tamerabassy@yahoo.com
}

\begin{abstract}
A new method for solving differential equations is introduced. The new method is called piecewise analytic method (PAM). The PAM is based on dividing the solution interval into subintervals. PAM gives an approximate analytic solution which is very accurate and can be applied to each subinterval successively. The piecewise analytic method can be made to be of any order of accuracy. PAM gives very interesting results especially when the solution contains singular points.
\end{abstract}

Keywords: Differential equation; Padé Approximants; Taylor series; Piecewise analytic method.

\section{Introduction:}

The piecewise analytic method (PAM) introduces a new treatment for solving differential equations. The PAM is based on dividing the solution interval into subintervals. Next, we obtain an approximate analytic solution which is very accurate and can be applied to each subinterval successively. The approximate analytic solution is based on truncated Taylor series [1,2] or Padé approximants [3-5]. In PAM, the solution accuracy can be controlled as needed. The PAM gives the exact solution in some special cases.

In "Piecewise Analytic Method", the word piecewise is used to describe a piecewise-defined solution that holds for each subinterval but may not hold for the whole domain of the differential equation; also, the word analytic is used because the solution has a valid power series expansion for any point in each subinterval.

In this paper, I will consider the basic initial value problem

$$
u^{\prime}=\phi(t, u), \quad u(0)=f_{0}, \quad t \geq 0 .
$$


which will be used for explaining the PAM and compare the obtained solution with the exact solutions. Equation (1) was solved numerically by Euler's method in 1768 [6-8]. Euler formula approximates $u_{n+1}$ by evaluating $\phi\left(t_{n}, u_{n}\right)$, that is, $\phi$ is evaluated at the single point $\left(t_{n}, u_{n}\right)$ but $\phi(t, u)$ is known at every point in the $(t, u)$ plane. C. Runge and W. Kutta make use of this fact around 1900 [9-11] and introduced Runge-Kutta method. The basic idea of Runge-Kutta method is to use information which is available, but has been neglected by Euler formula.

The drawback in the old methods is the lake of adequate control of the accuracy of the results and the local error due to truncation. The reason for this lack of accuracy in standard numerical methods is the short lengths of the equivalent power series. For example, a fourth order Runge-Kutta method is equivalent to a Taylor series with only five terms obtained by PAM.

In the following sections, I'll explain the piecewise analytic method and then apply the method to some illustrative examples which shows how much the piecewise analytic method is effective.

\section{Piecewise Analytic Method}

The piecewise analytic method (PAM) is divided into 3 steps. For explaining the steps, consider problem $(1)$

$$
u^{\prime}=\phi(t, u), \quad u(0)=f_{0}, \quad t \geq 0 .
$$

\section{The $1^{\text {st }}$ step:}

Since a computer cannot calculate indefinitely, let $0 \leq t$ be replaced by $0 \leq t \leq b$, in which $b$ is a positive constant. The value of $b$ is usually determined by the physics of the phenomenon under consideration. If the phenomenon occurs over a short period of time then $b$ can be chosen to be relatively small. If the phenomenon is long lasting then $b$ must be relatively large. In either case, $b$ is a fixed positive constant. The interval $0 \leq t \leq b$ is then divided into $n$ equal parts, each of length $h$, by the points $t_{i}=i h, i=0,1,2, \ldots, n$. The value $h=b / n$ is called the subinterval length. The points $t_{i}$ are called interval points see Figure 1 . $U_{n}$ denotes to the approximate analytic solution in the $n^{\text {th }}$ subinterval.

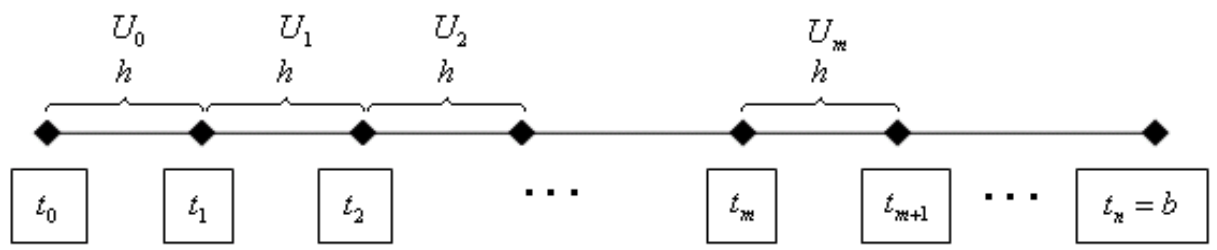

The $2^{\text {nd }}$ step:

Figure 1. 
In this step, we are concerned with obtaining the very accurate approximate analytic solution formula $U_{m}$ which can be applied to any subinterval $m\left(t \in\left[t_{m}, t_{m+1}\right], \quad m=0,1,2, \ldots,(n-1)\right)$.

The first order initial value problem

$$
u^{\prime}=\phi(t, u), \quad u(0)=f_{0}, \quad t \geq 0 .
$$

can be written in the form

$$
\begin{gathered}
\frac{d U_{m}}{d t}=\phi\left(t, U_{m}\right), \\
U_{m}\left(t_{m}\right)=f_{m}, \quad t \in\left[t_{m}, t_{m+1}\right], \quad m=0,1,2, \ldots,(n-1) .
\end{gathered}
$$

$U_{m}$ is used to identify the solution in the $m^{\text {th }}$ subinterval $\left[t_{m}, t_{m+1}\right]$ which is calculated successively and the initial value $U_{m}\left(t_{m}\right)=f_{m}$ is calculated by $f_{m}=U_{m-1}\left(t_{m}\right)$, where $U_{-1}\left(t_{0}\right)=f_{0}$.

The approximate analytic solution formula $U_{m}$ is calculated once and then it is applied to each subinterval successively.

Currently, I have two forms of the approximate solutions, one is the truncated Taylor series solution and the other is the Padé approximants solution.

In the case of truncated Taylor solution, $U_{m}$ is defined according to the needed accuracy. If we need the accuracy to be of $O\left(h^{s}\right), U_{m}$ will take the form

$$
U_{m}(t)=\sum_{n=0}^{s-1} c_{n}\left(t-t_{m}\right)^{n}=\sum_{n=0}^{s-1} \frac{\left(t-t_{m}\right)^{n}}{n !}\left(\left.\frac{d U_{m}}{d t}\right|_{t=t_{m}}\right) \quad t \in\left[t_{m}, t_{m+1}\right]
$$

In the case of Padé approximants solution[3-5], $U_{m}$ will take the form

$$
U_{m}(t)=\frac{P_{l}}{Q_{k}}=\frac{\sum_{n=0}^{l} p_{n}\left(t-t_{m}\right)^{n}}{\sum_{n=0}^{k} q_{n}\left(t-t_{m}\right)^{n}} \quad \text { where } l+k=s-1, \quad t \in\left[t_{m}, t_{m+1}\right]
$$

if we need the accuracy to be of $O\left(h^{s}\right)$.

Other approximate solution is under study.

\section{The $3^{\text {rd }}$ step:}

In this step we apply the approximate analytic solution formula, which is obtained in step 2, to each subinterval successively with initial values $f_{m}=U_{m-1}\left(t_{m}\right), U_{-1}\left(t_{0}\right)=f_{0}$.

Notes:

- There exist many methods for calculating (3). The first method, which I prefer, is the substitution by $U_{m}(t)=\sum_{n=0}^{s} c_{n}\left(t-t_{m}\right)^{n}$ and its derivatives into the ODE, then equating the coefficients of each power of $\left(t-t_{m}\right)^{n}$ sum to 
zero to get a recurrence relation. The recurrence relation expresses a coefficient $c_{n}$ in terms of the coefficients $c_{m}$ where $m<n$. The second method is calculating $U_{m}(t)=\sum_{n=0}^{s} \frac{\left(t-t_{m}\right)^{n}}{n !}\left(\left.\frac{d U_{m}}{d t}\right|_{t=t_{m}}\right)$ which is based on the manipulation of the function formula by classical differential calculus techniques. The other methods are based the new methods which give the truncated series solution like Adomian decomposition method[12], Improved Adomian decomposition method[13, 14], modified variational iteration method[15, 16], homotopy perturbation method[17], homotopy analysis method[18] and others, The results are constants that represent the value of derivatives at the point of evaluation.

- The PAM gives the exact solution in two cases:

1. If the exact solution is a polynomial with order $w$ and the truncated series approximation (3) is used with $s-1 \geq w$.

2. If the exact solution is a rational function $\frac{\sum_{n=0}^{z} p_{n}\left(t-t_{m}\right)^{n}}{\sum_{n=0}^{w} q_{n}\left(t-t_{m}\right)^{n}}$ and the Padé approximants (4) is used with $l \geq z$ and $k \geq w$.

- The truncated series (3) is suitable if the solution has no poles and bounded, if not, the Padé approximants (4) is more suitable than truncated series (3).

- I don’t know the best form for Padé approximants (4) but by experience I prefer $l=k=$ even .

- The Padé approximants coefficients $p_{n}(n=0,1,2, \ldots, l)$ and $q_{n}(0,1,2, \ldots, k)$ are determined by [3-5]

$$
\sum_{n=0}^{s \geq l+k} c_{n}\left(t-t_{m}\right)^{n}-\frac{\sum_{n=0}^{l} p_{n}\left(t-t_{m}\right)^{n}}{\sum_{n=0}^{k} q_{n}\left(t-t_{m}\right)^{n}}=O\left(\left(t-t_{m}\right)^{l+k+1}\right),
$$

setting $q_{0}=1$ and multiply (5) by $\sum_{n=0}^{k} q_{n}\left(t-t_{m}\right)^{n}$, which linearizes the equations coefficient. It can be written out in more detail as

$$
\left.\begin{array}{cc}
c_{l+1}+c_{l} q_{1}+\cdots+c_{l-k+1} q_{k} & =0 \\
c_{l+2}+c_{l+1} q_{1}+\cdots+c_{l-k+2} q_{k} & =0 \\
& \vdots \\
c_{l+k}+c_{l+k-1} q_{1}+\cdots+c_{l} q_{k} & =0
\end{array}\right\}
$$




$$
\left.\begin{array}{ll}
c_{0} & =p_{0} \\
c_{1}+c_{0} q_{1} & =p_{1} \\
c_{2}+c_{1} q_{1}+c_{0} q_{2} & =p_{2} \\
& \vdots \\
c_{l}+c_{l-1} q_{1}+\cdots+c_{0} q_{l} & =p_{l}
\end{array}\right\}
$$

Once, the $q$ 's are known from equations (6), equations (7) can be solved easily. If equations (6) and (7) are nonsingular, then they can be solved directly as follows;

$$
U_{m}(t)=\frac{\operatorname{det}\left|\begin{array}{cccc}
c_{l-k+1} & c_{l-k+2} & \cdots & c_{l+1} \\
\vdots & \vdots & \ddots & \vdots \\
c_{l} & c_{l+1} & \cdots & c_{l+k} \\
\sum_{j=k}^{l} c_{j-k} t^{j} & \sum_{j=k-1}^{l} c_{j-k+1} t^{j} & \cdots & \sum_{j=0}^{l} c_{j} t^{j}
\end{array}\right|}{\operatorname{det}\left|\begin{array}{cccc}
c_{l-k+1} & c_{l-k+2} & \cdots & c_{l+1} \\
\vdots & \vdots & \ddots & \vdots \\
c_{l} & c_{l+1} & \cdots & c_{l+k} \\
t^{k} & t^{k-1} & \cdots & 1
\end{array}\right|}
$$

For $l=1$ and $k=1$

$$
\begin{array}{ll}
p_{0}=c_{0}, & q_{0}=1, \\
p_{1}=\frac{c_{1}^{2}-c_{0} c_{2}}{c_{1}}, & q_{1}=-\frac{c_{2}}{c_{1}} .
\end{array}
$$

For $l=1$ and $k=2$

$$
\begin{array}{ll}
p_{0}=c_{0}, & q_{0}=1, \\
p_{1}=\frac{c_{1}^{3}-2 c_{0} c_{1} c_{2}+c_{0}{ }^{2} c_{3}}{c_{1}^{2}-c_{1} c_{2}}, & q_{1}=\frac{-c_{1} c_{2}+c_{0} c_{3}}{c_{1}^{2}-c_{0} c_{2}}, \\
q_{2}=\frac{c_{2}^{2}-c_{1} c_{3}}{c_{1}^{2}-c_{0} c_{2}} .
\end{array}
$$

For $l=2$ and $k=1$

$$
\begin{array}{ll}
p_{0}=c_{0}, & q_{0}=1, \\
p_{1}=\frac{c_{1} c_{2}-c_{0} c_{3}}{c_{2}}, & q_{1}=\frac{-c_{3}}{c_{2}} . \\
P_{2}=\frac{c_{2}^{2}-c_{1} c_{3}}{c_{2}}, &
\end{array}
$$

For $l=2$ and $k=2$ 


$$
\begin{aligned}
& p_{0}=c_{0}, \quad q_{0}=1 \text {, } \\
& p_{1}=\frac{c_{1} C_{2}^{2}-c_{1}{ }^{2} C_{3}-c_{0} c_{2} C_{3}+c_{0} c_{1} c_{4}}{c_{2}{ }^{2}-c_{1} C_{3}}, \quad q_{1}=\frac{-C_{2} C_{3}+c_{1} C_{4}}{c_{2}{ }^{2}-c_{1} C_{3}} \text {, } \\
& P_{2}=\frac{c_{2}^{3}-2 c_{1} C_{2} C_{3}+c_{0} c_{3}{ }^{2}+c_{1}{ }^{2} c_{4}-c_{0} c_{2} c_{4}}{c_{2}{ }^{2}-c_{1} c_{3}}, \quad q_{2}=\frac{c_{3}{ }^{2}-c_{2} c_{4}}{c_{2}{ }^{2}-c_{1} c_{3}} .
\end{aligned}
$$

For $l=3$ and $k=3$

$$
\begin{gathered}
P_{0}=c_{0}, \\
P_{1}=\left(-c_{1} c_{3}^{3}+2 c_{1} c_{2} c_{3} c_{4}+c_{0} c_{3}^{2} c_{4}-c_{1}^{2} c_{4}^{2}-c_{0} c_{2} c_{4}^{2}-c_{1} c_{2}^{2} c_{5}+c_{1}^{2} c_{3} c_{5}-c_{0} c_{2} c_{3} c_{5}+\right. \\
\left.c_{0} c_{1} c_{4} c_{5}+c_{0} c_{2}^{2} c_{6}-c_{0} c_{1} c_{3} c_{6}\right) /\left(-c_{3}^{3}+2 c_{2} c_{3} c_{4}-c_{1} c_{4}^{2}-c_{2}^{2} c_{5}+c_{1} c_{3} c_{5}\right), \\
P_{2}=\left(c_{2} c_{3}^{3}-2 c_{2}^{2} c_{3} c_{4}-c_{1} c_{3}^{2} c_{4}+2 c_{1} c_{2} c_{4}^{2}+c_{0} c_{3} c_{4}^{2}+c_{2}^{3} c_{5}-c_{0} c_{3}^{2} c_{5}-c_{1}^{2} c_{4} c_{5}-\right. \\
\left.\quad c_{0} c_{2} c_{4} c_{5}+c_{0} c_{1} c_{5}^{2}-c_{1} c_{2}^{2} c_{6}+c_{1}^{2} c_{3} c_{6}+c_{0} c_{2} c_{3} c_{6}-c_{0} c_{1} c_{4} c_{6}\right) / \\
\left(c_{3}^{3}-2 c_{2} c_{3} c_{4}+c_{1} c_{4}^{2}+c_{2}^{2} c_{5}-c_{1} c_{3} c_{5}\right), \\
P_{3}= \\
\left(c_{3}^{4}-3 c_{2} c_{3}^{2} c_{4}+c_{2}^{2} c_{4}^{2}+2 c_{1} c_{3} c_{4}^{2}-c_{0} c_{4}^{3}+2 c_{2}^{2} c_{3} c_{5}-2 c_{1} c_{3}^{2} c_{5}-2 c_{1} c_{2} c_{4} c_{5}+\right. \\
\quad 2 c_{0} c_{3} c_{4} c_{5}+c_{1}^{2} c_{5}^{2}-c_{0} c_{2} c_{5}^{2}-c_{2}^{3} c_{6}+2 c_{1} c_{2} c_{3} c_{6}-c_{0} c_{3}^{2} c_{6}- \\
\left.\quad c_{1}^{2} c_{4} c_{6}+c_{0} c_{2} c_{4} c_{6}\right) /\left(c_{3}^{3}-2 c_{2} c_{3} c_{4}+c_{1} c_{4}^{2}+c_{2}^{2} c_{5}-c_{1} c_{3} c_{5}\right), \\
q_{0}=1,
\end{gathered}
$$$$
q_{1}=\frac{-c_{3}^{2} c_{4}+c_{2} c_{4}^{2}+c_{2} c_{3} c_{5}-c_{1} c_{4} c_{5}-c_{2}^{2} c_{6}+c_{1} c_{3} c_{6}}{c_{3}^{3}-2 c_{2} c_{3} c_{4}+c_{1} c_{4}^{2}+c_{2}^{2} c_{5}-c_{1} c_{3} c_{5}},
$$$$
q_{2}=\frac{c_{3} c_{4}^{2}-c_{3}^{2} c_{5}-c_{2} c_{4} c_{5}+c_{1} c_{5}^{2}+c_{2} c_{3} c_{6}-c_{1} c_{4} c_{6}}{c_{3}^{3}-2 c_{2} c_{3} c_{4}+c_{1} c_{4}^{2}+c_{2}^{2} c_{5}-c_{1} c_{3} c_{5}} \text {, }
$$$$
q_{3}=\frac{-c_{4}^{3}+2 c_{3} c_{4} c_{5}-c_{2} c_{5}^{2}-c_{3}^{2} c_{6}+c_{2} c_{4} c_{6}}{c_{3}^{3}-2 c_{2} c_{3} c_{4}+c_{1} c_{4}^{2}+c_{2}^{2} c_{5}-c_{1} c_{3} c_{5}},
$$

For $l=4$ and $k=4$

$$
\begin{aligned}
P_{0}= & c_{0}, \\
P_{1}= & \left(-c_{1} c_{4}^{4}+3 c_{1} c_{3} c_{4}^{2} c_{5}+c_{0} c_{4}^{3} c_{5}-c_{1} c_{3}^{2} c_{5}^{2}-2 c_{1} c_{2} c_{4} c_{5}^{2}-2 c_{0} c_{3} c_{4} c_{5}^{2}+\right. \\
& c_{1}^{2} c_{5}^{3}+c_{0} c_{2} c_{5}^{3}-2 c_{1} c_{3}^{2} c_{4} c_{6}+2 c_{1} c_{2} c_{4}^{2} c_{6}-c_{0} c_{3} c_{4}^{2} c_{6}+ \\
& 2 c_{1} c_{2} c_{3} c_{5} c_{6}+2 c_{0} c_{3}^{2} c_{5} c_{6}-2 c_{1}^{2} c_{4} c_{5} c_{6}-c_{0} c_{1} c_{5}^{2} c_{6}-c_{1} c_{2}^{2} c_{6}^{2}+ \\
& c_{1}^{2} c_{3} c_{6}^{2}-c_{0} c_{2} c_{3} c_{6}^{2}+c_{0} c_{1} c_{4} c_{6}^{2}+c_{1} c_{3}^{3} c_{7}-2 c_{1} c_{2} c_{3} c_{4} c_{7}+ \\
& c_{0} c_{3}^{2} c_{4} c_{7}+c_{1}^{2} c_{4}^{2} c_{7}-c_{0} c_{2} c_{4}^{2} c_{7}+c_{1} c_{2}^{2} c_{5} c_{7}-c_{1}^{2} c_{3} c_{5} c_{7}- \\
& c_{0} c_{2} c_{3} c_{5} c_{7}+c_{0} c_{1} c_{4} c_{5} c_{7}+c_{0} c_{2}^{2} c_{6} c_{7}-c_{0} c_{1} c_{3} c_{6} c_{7}-
\end{aligned}
$$




$$
\begin{aligned}
& \left.c_{0} c_{3}^{3} c_{8}+2 c_{0} c_{2} c_{3} c_{4} c_{8}-c_{0} c_{1} c_{4}^{2} c_{8}-c_{0} c_{2}^{2} c_{5} c_{8}+c_{0} c_{1} c_{3} c_{5} c_{8}\right) / \\
& \left(-c_{4}^{4}+3 c_{3} c_{4}^{2} c_{5}-c_{3}^{2} c_{5}^{2}-2 c_{2} c_{4} c_{5}^{2}+c_{1} c_{5}^{3}-2 c_{3}^{2} c_{4} c_{6}+\right. \\
& 2 c_{2} c_{4}^{2} c_{6}+2 c_{2} c_{3} c_{5} c_{6}-2 c_{1} c_{4} c_{5} c_{6}-c_{2}^{2} c_{6}^{2}+c_{1} c_{3} c_{6}^{2}+ \\
& \left.c_{3}^{3} c_{7}-2 c_{2} c_{3} c_{4} c_{7}+c_{1} c_{4}^{2} c_{7}+c_{2}^{2} c_{5} c_{7}-c_{1} c_{3} c_{5} c_{7}\right) \text {, } \\
& P_{2}= \\
& \left(-c_{2} c_{4}^{4}+3 c_{2} c_{3} c_{4}^{2} c_{5}+c_{1} c_{4}^{3} c_{5}-c_{2} c_{3}^{2} c_{5}^{2}-2 c_{2}^{2} c_{4} c_{5}^{2}-2 c_{1} c_{3} c_{4} c_{5}^{2}-c_{0} c_{4}^{2} c_{5}^{2}+\right. \\
& 2 c_{1} c_{2} c_{5}^{3}+c_{0} c_{3} c_{5}^{3}-2 c_{2} c_{3}^{2} c_{4} c_{6}+2 c_{2}^{2} c_{4}^{2} c_{6}-c_{1} c_{3} c_{4}^{2} c_{6}+c_{0} c_{4}^{3} c_{6}+ \\
& 2 c_{2}^{2} c_{3} c_{5} c_{6}+2 c_{1} c_{3}^{2} c_{5} c_{6}-2 c_{1} c_{2} c_{4} c_{5} c_{6}-c_{1}^{2} c_{5}^{2} c_{6}-c_{0} c_{2} c_{5}^{2} c_{6}- \\
& c_{2}^{3} c_{6}^{2}+c_{1}^{2} c_{4} c_{6}^{2}-c_{0} c_{2} c_{4} c_{6}^{2}+c_{0} c_{1} c_{5} c_{6}^{2}+c_{2} c_{3}^{3} c_{7}-2 c_{2}^{2} c_{3} c_{4} c_{7}+ \\
& c_{1} c_{3}^{2} c_{4} c_{7}-c_{0} c_{3} c_{4}^{2} c_{7}+c_{2}^{3} c_{5} c_{7}-2 c_{1} c_{2} c_{3} c_{5} c_{7}-c_{0} c_{3}^{2} c_{5} c_{7}+ \\
& c_{1}^{2} c_{4} c_{5} c_{7}+3 c_{0} c_{2} c_{4} c_{5} c_{7}-c_{0} c_{1} c_{5}^{2} c_{7}+c_{1} c_{2}^{2} c_{6} c_{7}-c_{1}^{2} c_{3} c_{6} c_{7}+ \\
& c_{0} c_{2} c_{3} c_{6} c_{7}-c_{0} c_{1} c_{4} c_{6} c_{7}-c_{0} c_{2}^{2} c_{7}^{2}+c_{0} c_{1} c_{3} c_{7}^{2}-c_{1} c_{3}^{3} c_{8}+ \\
& 2 c_{1} c_{2} c_{3} c_{4} c_{8}+c_{0} c_{3}^{2} c_{4} c_{8}-c_{1}^{2} c_{4}^{2} c_{8}-c_{0} c_{2} c_{4}^{2} c_{8}-c_{1} c_{2}^{2} c_{5} c_{8}+ \\
& \left.c_{1}^{2} c_{3} c_{5} c_{8}-c_{0} c_{2} c_{3} c_{5} c_{8}+c_{0} c_{1} c_{4} c_{5} c_{8}+c_{0} c_{2}^{2} c_{6} c_{8}-c_{0} c_{1} c_{3} c_{6} c_{8}\right) / \\
& \left(-c_{4}^{4}+3 c_{3} c_{4}^{2} c_{5}-c_{3}^{2} c_{5}^{2}-2 c_{2} c_{4} c_{5}^{2}+c_{1} c_{5}^{3}-2 c_{3}^{2} c_{4} c_{6}+\right. \\
& 2 c_{2} c_{4}^{2} c_{6}+2 c_{2} c_{3} c_{5} c_{6}-2 c_{1} c_{4} c_{5} c_{6}-c_{2}^{2} c_{6}^{2}+c_{1} c_{3} c_{6}^{2}+ \\
& \left.c_{3}^{3} c_{7}-2 c_{2} c_{3} c_{4} c_{7}+c_{1} c_{4}^{2} c_{7}+c_{2}^{2} c_{5} c_{7}-c_{1} c_{3} c_{5} c_{7}\right) \text {, } \\
& P_{3}= \\
& \left(-c_{3} c_{4}^{4}+3 c_{3}^{2} c_{4}^{2} c_{5}+c_{2} c_{4}^{3} c_{5}-c_{3}^{3} c_{5}^{2}-4 c_{2} c_{3} c_{4} c_{5}^{2}-c_{1} c_{4}^{2} c_{5}^{2}+c_{2}^{2} c_{5}^{3}+2 c_{1} c_{3} c_{5}^{3}+\right. \\
& c_{0} c_{4} c_{5}^{3}-2 c_{3}^{3} c_{4} c_{6}+c_{2} c_{3} c_{4}^{2} c_{6}+c_{1} c_{4}^{3} c_{6}+4 c_{2} c_{3}^{2} c_{5} c_{6}- \\
& 2 c_{1} c_{3} c_{4} c_{5} c_{6}-2 c_{0} c_{4}^{2} c_{5} c_{6}-2 c_{1} c_{2} c_{5}^{2} c_{6}-c_{0} c_{3} c_{5}^{2} c_{6}-2 c_{2}^{2} c_{3} c_{6}^{2}+ \\
& c_{1} c_{3}^{2} c_{6}^{2}+2 c_{0} c_{3} c_{4} c_{6}^{2}+c_{1}^{2} c_{5} c_{6}^{2}+c_{0} c_{2} c_{5} c_{6}^{2}-c_{0} c_{1} c_{6}^{3}+c_{3}^{4} c_{7}- \\
& c_{2} c_{3}^{2} c_{4} c_{7}-c_{2}^{2} c_{4}^{2} c_{7}+c_{0} c_{4}^{3} c_{7}-2 c_{1} c_{3}^{2} c_{5} c_{7}+4 c_{1} c_{2} c_{4} c_{5} c_{7}- \\
& c_{1}^{2} c_{5}^{2} c_{7}-c_{0} c_{2} c_{5}^{2} c_{7}+c_{2}^{3} c_{6} c_{7}-c_{0} c_{3}^{2} c_{6} c_{7}-c_{1}^{2} c_{4} c_{6} c_{7}- \\
& c_{0} c_{2} c_{4} c_{6} c_{7}+2 c_{0} c_{1} c_{5} c_{6} c_{7}-c_{1} c_{2}^{2} c_{7}^{2}+c_{1}^{2} c_{3} c_{7}^{2}+c_{0} c_{2} c_{3} c_{7}^{2}- \\
& c_{0} c_{1} c_{4} c_{7}^{2}-c_{2} c_{3}^{3} c_{8}+2 c_{2}^{2} c_{3} c_{4} c_{8}+c_{1} c_{3}^{2} c_{4} c_{8}-2 c_{1} c_{2} c_{4}^{2} c_{8}- \\
& c_{0} c_{3} c_{4}^{2} c_{8}-c_{2}^{3} c_{5} c_{8}+c_{0} c_{3}^{2} c_{5} c_{8}+c_{1}^{2} c_{4} c_{5} c_{8}+c_{0} c_{2} c_{4} c_{5} c_{8}- \\
& \left.c_{0} c_{1} c_{5}^{2} c_{8}+c_{1} c_{2}^{2} c_{6} c_{8}-c_{1}^{2} c_{3} c_{6} c_{8}-c_{0} c_{2} c_{3} c_{6} c_{8}+c_{0} c_{1} c_{4} c_{6} c_{8}\right) / \\
& \left(-c_{4}^{4}+3 c_{3} c_{4}^{2} c_{5}-c_{3}^{2} c_{5}^{2}-2 c_{2} c_{4} c_{5}^{2}+c_{1} c_{5}^{3}-2 c_{3}^{2} c_{4} c_{6}+\right. \\
& 2 c_{2} c_{4}^{2} c_{6}+2 c_{2} c_{3} c_{5} c_{6}-2 c_{1} c_{4} c_{5} c_{6}-c_{2}^{2} c_{6}^{2}+c_{1} c_{3} c_{6}^{2}+ \\
& \left.c_{3}^{3} c_{7}-2 c_{2} c_{3} c_{4} c_{7}+c_{1} c_{4}^{2} c_{7}+c_{2}^{2} c_{5} c_{7}-c_{1} c_{3} c_{5} c_{7}\right) \text {, }
\end{aligned}
$$




$$
\begin{aligned}
& P_{4}=\left(c_{4}^{5}-4 c_{3} c_{4}^{3} c_{5}+3 c_{3}^{2} c_{4} c_{5}^{2}+3 c_{2} c_{4}^{2} c_{5}^{2}-2 c_{2} c_{3} c_{5}^{3}-2 c_{1} c_{4} c_{5}^{3}+c_{0} c_{5}^{4}+\right. \\
& 3 c_{3}^{2} c_{4}^{2} c_{6}-3 c_{2} c_{4}^{3} c_{6}-2 c_{3}^{3} c_{5} c_{6}-2 c_{2} c_{3} c_{4} c_{5} c_{6}+4 c_{1} c_{4}^{2} c_{5} c_{6}+ \\
& c_{2}^{2} c_{5}^{2} c_{6}+2 c_{1} c_{3} c_{5}^{2} c_{6}-3 c_{0} c_{4} c_{5}^{2} c_{6}+c_{2} c_{3}^{2} c_{6}^{2}+2 c_{2}^{2} c_{4} c_{6}^{2}- \\
& 4 c_{1} c_{3} c_{4} c_{6}^{2}+c_{0} c_{4}^{2} c_{6}^{2}-2 c_{1} c_{2} c_{5} c_{6}^{2}+2 c_{0} c_{3} c_{5} c_{6}^{2}+c_{1}^{2} c_{6}^{3}-c_{0} c_{2} c_{6}^{3}- \\
& 2 c_{3}^{3} c_{4} c_{7}+4 c_{2} c_{3} c_{4}^{2} c_{7}-2 c_{1} c_{4}^{3} c_{7}+2 c_{2} c_{3}^{2} c_{5} c_{7}-4 c_{2}^{2} c_{4} c_{5} c_{7}+ \\
& 2 c_{0} c_{4}^{2} c_{5} c_{7}+2 c_{1} c_{2} c_{5}^{2} c_{7}-2 c_{0} c_{3} c_{5}^{2} c_{7}-2 c_{2}^{2} c_{3} c_{6} c_{7}+2 c_{1} c_{3}^{2} c_{6} c_{7}+ \\
& 2 c_{1} c_{2} c_{4} c_{6} c_{7}-2 c_{0} c_{3} c_{4} c_{6} c_{7}-2 c_{1}^{2} c_{5} c_{6} c_{7}+2 c_{0} c_{2} c_{5} c_{6} c_{7}+ \\
& c_{2}^{3} c_{7}^{2}-2 c_{1} c_{2} c_{3} c_{7}^{2}+c_{0} c_{3}^{2} c_{7}^{2}+c_{1}^{2} c_{4} c_{7}^{2}-c_{0} c_{2} c_{4} c_{7}^{2}+c_{3}^{4} c_{8}- \\
& 3 c_{2} c_{3}^{2} c_{4} c_{8}+c_{2}^{2} c_{4}^{2} c_{8}+2 c_{1} c_{3} c_{4}^{2} c_{8}-c_{0} c_{4}^{3} c_{8}+2 c_{2}^{2} c_{3} c_{5} c_{8}- \\
& 2 c_{1} c_{3}^{2} c_{5} c_{8}-2 c_{1} c_{2} c_{4} c_{5} c_{8}+2 c_{0} c_{3} c_{4} c_{5} c_{8}+c_{1}^{2} c_{5}^{2} c_{8}-c_{0} c_{2} c_{5}^{2} c_{8}- \\
& \left.c_{2}^{3} c_{6} c_{8}+2 c_{1} c_{2} c_{3} c_{6} c_{8}-c_{0} c_{3}^{2} c_{6} c_{8}-c_{1}^{2} c_{4} c_{6} c_{8}+c_{0} c_{2} c_{4} c_{6} c_{8}\right) / \\
& \left(c_{4}^{4}-3 c_{3} c_{4}^{2} c_{5}+c_{3}^{2} c_{5}^{2}+2 c_{2} c_{4} c_{5}^{2}-c_{1} c_{5}^{3}+2 c_{3}^{2} c_{4} c_{6}-\right. \\
& 2 c_{2} c_{4}^{2} c_{6}-2 c_{2} c_{3} c_{5} c_{6}+2 c_{1} c_{4} c_{5} c_{6}+c_{2}^{2} c_{6}^{2}-c_{1} c_{3} c_{6}^{2}- \\
& \left.c_{3}^{3} c_{7}+2 c_{2} c_{3} c_{4} c_{7}-c_{1} c_{4}^{2} c_{7}-c_{2}^{2} c_{5} c_{7}+c_{1} c_{3} c_{5} c_{7}\right) \text {, } \\
& q_{0}=1 \text {, } \\
& q_{1}=\left(-c_{4}^{3} c_{5}+2 c_{3} c_{4} c_{5}^{2}-c_{2} c_{5}^{3}+c_{3} c_{4}^{2} c_{6}-2 c_{3}^{2} c_{5} c_{6}+c_{1} c_{5}^{2} c_{6}+c_{2} c_{3} c_{6}^{2}-\right. \\
& c_{1} c_{4} c_{6}^{2}-c_{3}^{2} c_{4} c_{7}+c_{2} c_{4}^{2} c_{7}+c_{2} c_{3} c_{5} c_{7}-c_{1} c_{4} c_{5} c_{7}-c_{2}^{2} c_{6} c_{7}+ \\
& \left.c_{1} c_{3} c_{6} c_{7}+c_{3}^{3} c_{8}-2 c_{2} c_{3} c_{4} c_{8}+c_{1} c_{4}^{2} c_{8}+c_{2}^{2} c_{5} c_{8}-c_{1} c_{3} c_{5} c_{8}\right) / \\
& \left(c_{4}^{4}-3 c_{3} c_{4}^{2} c_{5}+c_{3}^{2} c_{5}^{2}+2 c_{2} c_{4} c_{5}^{2}-c_{1} c_{5}^{3}+2 c_{3}^{2} c_{4} c_{6}-\right. \\
& 2 c_{2} c_{4}^{2} c_{6}-2 c_{2} c_{3} c_{5} c_{6}+2 c_{1} c_{4} c_{5} c_{6}+c_{2}^{2} c_{6}^{2}-c_{1} c_{3} c_{6}^{2}- \\
& \left.c_{3}^{3} c_{7}+2 c_{2} c_{3} c_{4} c_{7}-c_{1} c_{4}^{2} c_{7}-c_{2}^{2} c_{5} c_{7}+c_{1} c_{3} c_{5} c_{7}\right) \text {, } \\
& q_{2}=\left(-c_{4}^{2} c_{5}^{2}+c_{3} c_{5}^{3}+c_{4}^{3} c_{6}-c_{2} c_{5}^{2} c_{6}-c_{2} c_{4} c_{6}^{2}+c_{1} c_{5} c_{6}^{2}-c_{3} c_{4}^{2} c_{7}-c_{3}^{2} c_{5} c_{7}+\right. \\
& 3 c_{2} c_{4} c_{5} c_{7}-c_{1} c_{5}^{2} c_{7}+c_{2} c_{3} c_{6} c_{7}-c_{1} c_{4} c_{6} c_{7}-c_{2}^{2} c_{7}^{2}+c_{1} c_{3} c_{7}^{2}+ \\
& \left.c_{3}^{2} c_{4} c_{8}-c_{2} c_{4}^{2} c_{8}-c_{2} c_{3} c_{5} c_{8}+c_{1} c_{4} c_{5} c_{8}+c_{2}^{2} c_{6} c_{8}-c_{1} c_{3} c_{6} c_{8}\right) / \\
& \left(-c_{4}^{4}+3 c_{3} c_{4}^{2} c_{5}-c_{3}^{2} c_{5}^{2}-2 c_{2} c_{4} c_{5}^{2}+c_{1} c_{5}^{3}-2 c_{3}^{2} c_{4} c_{6}+\right. \\
& 2 c_{2} c_{4}^{2} c_{6}+2 c_{2} c_{3} c_{5} c_{6}-2 c_{1} c_{4} c_{5} c_{6}-c_{2}^{2} c_{6}^{2}+c_{1} c_{3} c_{6}^{2}+ \\
& \left.c_{3}^{3} c_{7}-2 c_{2} c_{3} c_{4} c_{7}+c_{1} c_{4}^{2} c_{7}+c_{2}^{2} c_{5} c_{7}-c_{1} c_{3} c_{5} c_{7}\right), \\
& q_{3}=\left(c_{4} c_{5}^{3}-2 c_{4}^{2} c_{5} c_{6}-c_{3} c_{5}^{2} c_{6}+2 c_{3} c_{4} c_{6}^{2}+c_{2} c_{5} c_{6}^{2}-c_{1} c_{6}^{3}+c_{4}^{3} c_{7}-c_{2} c_{5}^{2} c_{7}-\right. \\
& c_{3}^{2} c_{6} c_{7}-c_{2} c_{4} c_{6} c_{7}+2 c_{1} c_{5} c_{6} c_{7}+c_{2} c_{3} c_{7}^{2}-c_{1} c_{4} c_{7}^{2}-c_{3} c_{4}^{2} c_{8}+ \\
& \left.c_{3}^{2} c_{5} c_{8}+c_{2} c_{4} c_{5} c_{8}-c_{1} c_{5}^{2} c_{8}-c_{2} c_{3} c_{6} c_{8}+c_{1} c_{4} c_{6} c_{8}\right) /
\end{aligned}
$$




$$
\begin{gathered}
\left(-c_{4}^{4}+3 c_{3} c_{4}^{2} c_{5}-c_{3}^{2} c_{5}^{2}-2 c_{2} c_{4} c_{5}^{2}+c_{1} c_{5}^{3}-2 c_{3}^{2} c_{4} c_{6}+\right. \\
2 c_{2} c_{4}^{2} c_{6}+2 c_{2} c_{3} c_{5} c_{6}-2 c_{1} c_{4} c_{5} c_{6}-c_{2}^{2} c_{6}^{2}+c_{1} c_{3} c_{6}^{2}+ \\
\left.c_{3}^{3} c_{7}-2 c_{2} c_{3} c_{4} c_{7}+c_{1} c_{4}^{2} c_{7}+c_{2}^{2} c_{5} c_{7}-c_{1} c_{3} c_{5} c_{7}\right), \\
q_{4}=\left(-c_{5}^{4}+3 c_{4} c_{5}^{2} c_{6}-c_{4}^{2} c_{6}^{2}-2 c_{3} c_{5} c_{6}^{2}+c_{2} c_{6}^{3}-2 c_{4}^{2} c_{5} c_{7}+\right. \\
2 c_{3} c_{5}^{2} c_{7}+2 c_{3} c_{4} c_{6} c_{7}-2 c_{2} c_{5} c_{6} c_{7}-c_{3}^{2} c_{7}^{2}+c_{2} c_{4} c_{7}^{2}+ \\
\left.c_{4}^{3} c_{8}-2 c_{3} c_{4} c_{5} c_{8}+c_{2} c_{5}^{2} c_{8}+c_{3}^{2} c_{6} c_{8}-c_{2} c_{4} c_{6} c_{8}\right) / \\
\left(-c_{4}^{4}+3 c_{3} c_{4}^{2} c_{5}-c_{3}^{2} c_{5}^{2}-2 c_{2} c_{4} c_{5}^{2}+c_{1} c_{5}^{3}-2 c_{3}^{2} c_{4} c_{6}+\right. \\
2 c_{2} c_{4}^{2} c_{6}+2 c_{2} c_{3} c_{5} c_{6}-2 c_{1} c_{4} c_{5} c_{6}-c_{2}^{2} c_{6}^{2}+c_{1} c_{3} c_{6}^{2}+ \\
\left.c_{3}^{3} c_{7}-2 c_{2} c_{3} c_{4} c_{7}+c_{1} c_{4}^{2} c_{7}+c_{2}^{2} c_{5} c_{7}-c_{1} c_{3} c_{5} c_{7}\right),
\end{gathered}
$$

And so on, where we can calculate any desired Padé approximants by using any symbolic mathematical program for any series $U_{m}(t)=\sum_{n=0}^{s} c_{n}\left(t-t_{m}\right)^{n}$ and then all what we will do is only substituting by $c_{n}{ }^{\prime} s$ in the suitable $p$ 's and $q$ 's for obtaining the desired Padé approximants .

\section{Illustrative Examples:}

In the following examples, I show how PAM can be used for solving ordinary differential equation (linear and non-linear). The results are very interesting.

\subsection{Illustrative Example 1:}

For illustrative purpose, consider the ordinary differential equation

$$
\frac{d u}{d t}=-u, \quad u(0)=1
$$

This is a linear problem and can be solved exactly to yield the solution

$$
u(t)=e^{-t}
$$

For solving (15) using PAM

First, fixing $b=10$ and $h=0.1$ which leads to

$$
n=\frac{b-t_{0}}{h}=\frac{10-0}{0.1}=100 \text { subintervals }
$$

Next, the $1^{\text {st }}$ order initial value problem (15) can be written in the form

$$
\begin{gathered}
\frac{d U_{m}}{d t}=-U_{m}, \quad U_{m}\left(t_{m}\right)=f_{m}, \quad t \in\left[t_{m}, t_{m+1}\right], \\
\text { where } f_{m}=U_{m-1}\left(t_{m}\right), U_{-1}\left(t_{0}\right)=1, \quad m=0,1,2, \ldots, n-1
\end{gathered}
$$

Equation (18) is valid for each subinterval so, I'll obtain a solution for (18) and consequently the solution will be valid for each subinterval. 
Substituting by $U_{m}(t)=\sum_{n=0}^{s} c_{n}\left(t-t_{m}\right)^{n}$ and its derivatives into (18) leads to

$$
\sum_{n=1}^{s} n c_{n}\left(t-t_{m}\right)^{n-1}=-\sum_{n=0}^{s} c_{n}\left(t-t_{m}\right)^{n}, \quad c_{0}=f_{m}, \quad t \in\left[t_{m}, t_{m+1}\right] .
$$

Solving (19) leads to:

$$
\begin{gathered}
c_{0}=f_{m}, \\
c_{1}=\frac{-c_{0}}{1}=-f_{m}, \\
c_{2}=\frac{-c_{1}}{2}=\frac{f_{m}}{2}, \\
c_{3}=\frac{-c_{2}}{3}=\frac{-f_{m}}{3 !}, \\
\vdots \\
c_{n}=\frac{-c_{n-1}}{n}=\frac{(-1)^{n} f_{m}}{n !},
\end{gathered}
$$

Therefore, if we need accuracy of $O\left(h^{5}\right)$ :

Substituting by (20) into (3), we get the approximate analytic Taylor series solution

$$
U_{m}(t) \simeq f_{m}\left(1-\left(t-t_{m}\right)+\frac{\left(t-t_{m}\right)^{2}}{2 !}-\frac{\left(t-t_{m}\right)^{3}}{3 !}+\frac{\left(t-t_{m}\right)^{4}}{4 !}\right), \quad t \in\left[t_{m}, t_{m+1}\right]
$$

and substitute by (20) into (12) for obtaining $p$ 's and $q$ 's and then substitute by them into (4), we get the approximate Padé solution

$$
U_{m}(t) \simeq \frac{f_{m}\left(12-6\left(t-t_{m}\right)+\left(t-t_{m}\right)^{2}\right)}{12+6\left(t-t_{m}\right)+\left(t-t_{m}\right)^{2}}, \quad t \in\left[t_{m}, t_{m+1}\right]
$$

If we need accuracy of $O\left(h^{9}\right)$ :

Following the same procedure, the approximate analytic Taylor series solution will take the form

$$
\begin{aligned}
U_{m}(t) \simeq & f_{m}\left(1-\left(t-t_{m}\right)+\frac{\left(t-t_{m}\right)^{2}}{2 !}-\frac{\left(t-t_{m}\right)^{3}}{3 !}+\frac{\left(t-t_{m}\right)^{4}}{4 !}-\frac{\left(t-t_{m}\right)^{5}}{5 !}+\right. \\
& \left.\frac{\left(t-t_{m}\right)^{6}}{6 !}-\frac{\left(t-t_{m}\right)^{7}}{7 !}+\frac{\left(t-t_{m}\right)^{8}}{8 !}\right), \quad t \in\left[t_{m}\right.
\end{aligned}
$$

and the approximate Padé approximants will take the form

$U_{m}(t) \simeq \frac{f_{m}\left(1680-840\left(t-t_{m}\right)+180\left(t-t_{m}\right)^{2}-20\left(t-t_{m}\right)^{3}+\left(t-t_{m}\right)^{4}\right)}{1680+840\left(t-t_{m}\right)+180\left(t-t_{m}\right)^{2}+20\left(t-t_{m}\right)^{3}+\left(t-t_{m}\right)^{4}}, t \in\left[t_{m}, t_{m+1}\right]$

According to the needed accuracy order, we will use the suitable form of analytic approximation. 
The next step is substituting by the desired approximate analytic solution in each subinterval successively. Figure 2 shows the PAM solution (24) using $h=0.1$. Table 1 summarizes the results and successive procedures for illustrative example 1. Table 2 shows figures of the absolute error between the exact solution (16) of the ordinary differential equation (15)and the truncated series solution (23) and Padé approximants solution $[4 / 4]$ (24), which both have $O\left(h^{9}\right)$ for different values of $h$. Table 3 and

Table 4 show figures of the absolute error between the exact solution of (15) and different forms of the truncated series and Padé approximants using $h=0.01$ and $h=1$ respectively.

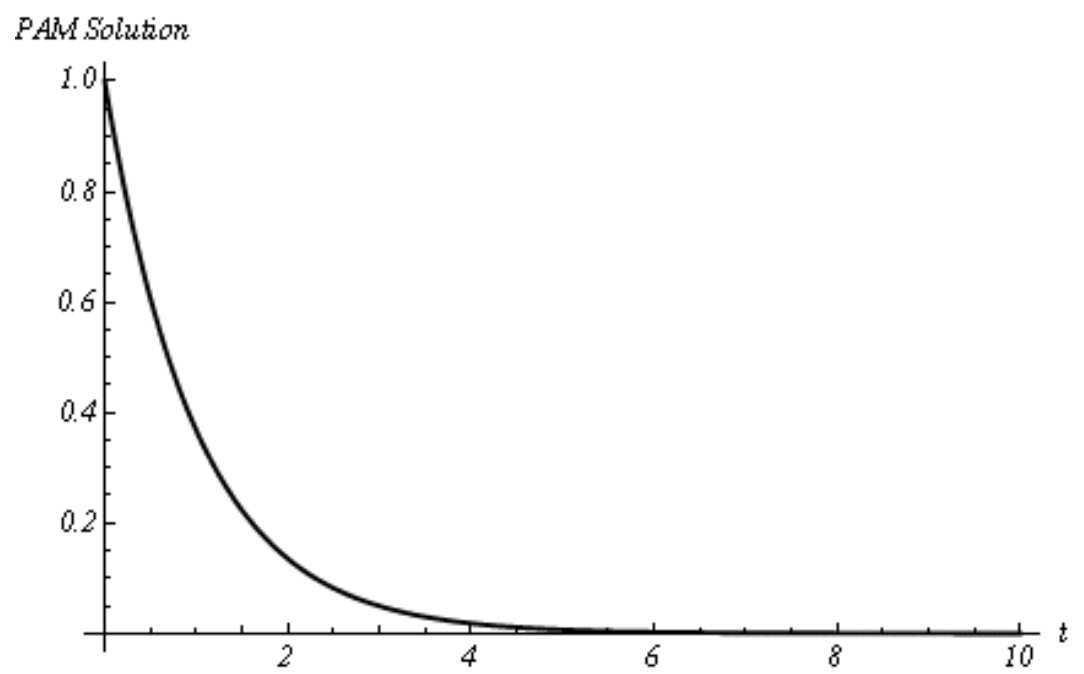

Figure 2: The PAM solution (24) of problem (15) using $h=0.1$.

Table 1.

\begin{tabular}{|c|c|c|c|}
\hline$m$ & $\begin{array}{l}\text { Interval= } \\
{\left[t_{m}, t_{m+1}\right]}\end{array}$ & $f_{m}=U_{m-1}\left(t_{m}\right)$ & $U_{m}(t)=$ \\
\hline 0 & $\begin{array}{l}{\left[t_{0}, t_{1}\right]=} \\
{[0.0,0.1]}\end{array}$ & $f_{0}=1$ & $\begin{array}{c}\text {----------accuracy } O\left(h^{4}\right) \text {-------- } \\
\text { Series }\end{array}$ \\
\hline 1 & $\begin{array}{l}{\left[t_{1}, t_{2}\right]=} \\
{[0.1,0.2]}\end{array}$ & $f_{1}=U_{0}(0.1)$ & $U_{m}(t) \simeq f_{m}\left(1-\left(t-t_{m}\right)+\frac{\left(t-t_{m}\right)^{2}}{2 !}-\right)$ \\
\hline 2 & $\begin{array}{l}{\left[t_{2}, t_{3}\right]=} \\
{[0.2,0.3]}\end{array}$ & $f_{2}=U_{1}(0.2)$ & $\left(\begin{array}{l}\frac{\left(t-t_{m}\right)^{\mathrm{S}}}{3 !}+\frac{\left(t-t_{m}\right)^{4}}{4 !} \\
\text { Padé }\end{array}\right.$ \\
\hline & $\begin{array}{l}{\left[t_{3}, t_{4}\right]=} \\
{[0.3,0.4]}\end{array}$ & $f_{3}=U_{2}(0.3)$ & $U_{m}(t) \simeq \frac{f_{m}\left(12-6\left(t-t_{m}\right)+\left(t-t_{m}\right)^{2}\right)}{12+6\left(t-t_{m}\right)+\left(t-t_{m}\right)^{2}}$ \\
\hline
\end{tabular}




\begin{tabular}{|c|c|c|c|}
\hline 4 & $\begin{array}{l}{\left[t_{4}, t_{5}\right]=} \\
{[0.4,0.5]}\end{array}$ & $f_{4}=U_{3}(0.4)$ & ---------accuracy $O\left(h^{9}\right)$--------- \\
\hline$\vdots$ & $\vdots$ & : & $U_{m}(t) \simeq f_{m}\left(1-\left(t-t_{m}\right)+\frac{\left(t-t_{m}\right)^{2}}{2 !}-\frac{\left(t-t_{m}\right)^{3}}{3 !}+\right.$ \\
\hline$r$ & $\begin{array}{c}{\left[t_{r}, t_{r+1}\right]=} \\
{[r h,(r+1) h]}\end{array}$ & $\begin{array}{l}f_{r}=U_{r-1}\left(t_{r}\right) \\
=U_{r-1}(r h)\end{array}$ & $\frac{\left(t-t_{m}\right)^{4}}{4 !}-\frac{\left(t-t_{m}\right)^{5}}{5 !}+\frac{\left(t-t_{m}\right)^{6}}{6 !}-$ \\
\hline$\vdots$ & $\vdots$ & $:$ & $\left.\frac{\left(t-t_{m}\right)^{7}}{7 !}+\frac{\left(t-t_{m}\right)^{8}}{8 !}\right)$ \\
\hline 99 & $\begin{array}{c}{\left[t_{99}, t_{100}\right]=} \\
{[9.9,10]}\end{array}$ & $f_{99}=U_{98}(9.9)$ & $\begin{array}{c}\text { Padé } \\
U_{m}(t) \simeq\left(f_{m}\left(1680-840\left(t-t_{m}\right)+180\left(t-t_{m}\right)^{2}-\right.\right. \\
\left.\left.20\left(t-t_{m}\right)^{3}+\left(t-t_{m}\right)^{4}\right)\right) /(1680+840(t- \\
\left.\left.t_{m}\right)+180\left(t-t_{m}\right)^{2}+20\left(t-t_{m}\right)^{3}+\left(t-t_{m}\right)^{4}\right),\end{array}$ \\
\hline
\end{tabular}

Table 2: Figures of the absolute error between the exact solution of the ordinary differential equation (15) and the truncated series solution (23) and Padé approximants solution $[4 / 4]$ (24) using different values of $h$.

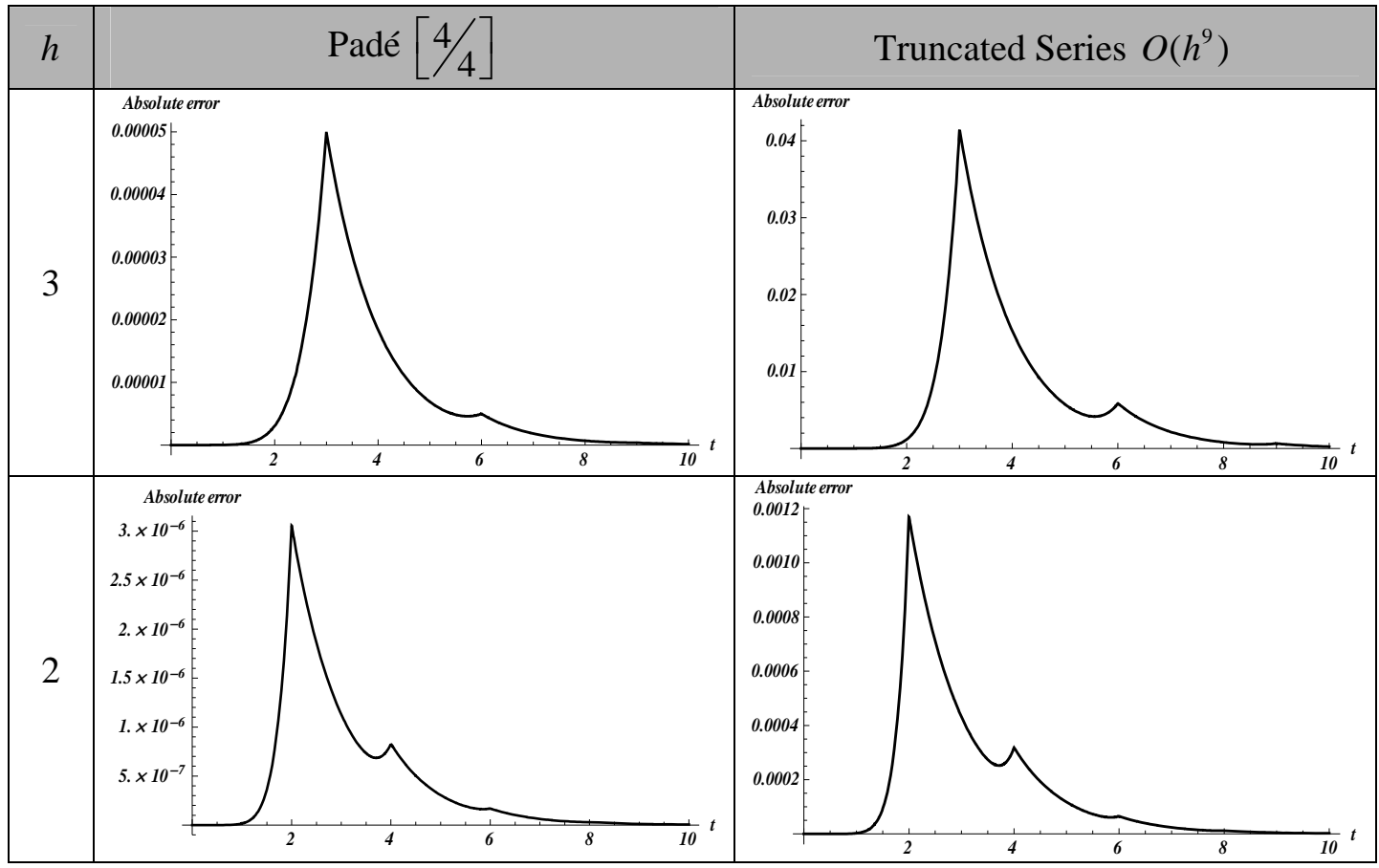


Piecwise Analytic Method

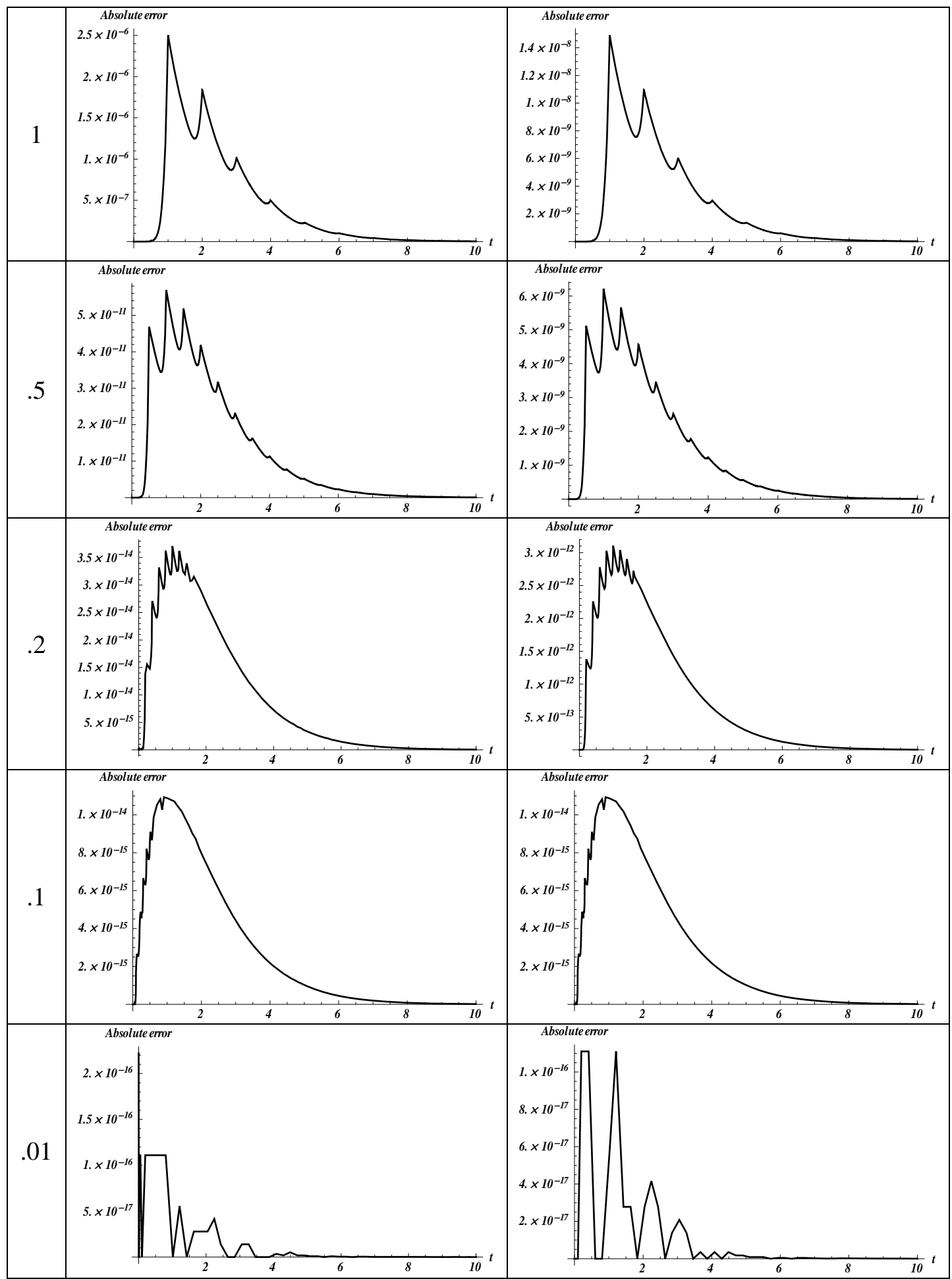


Table 3: Figures of the absolute error between the exact solution of (15) and different forms of the truncated series and Padé approximants using $h=0.01$.

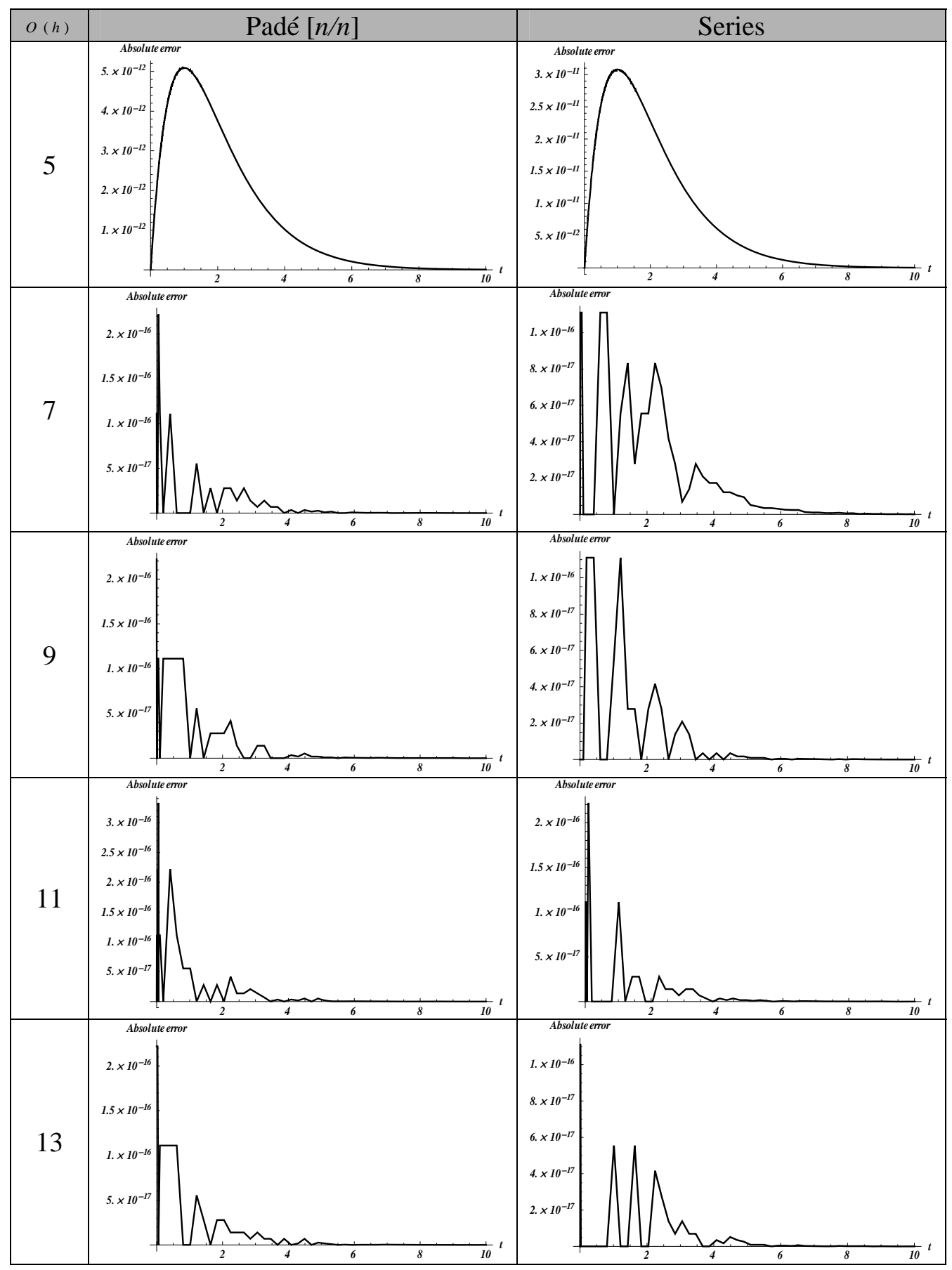




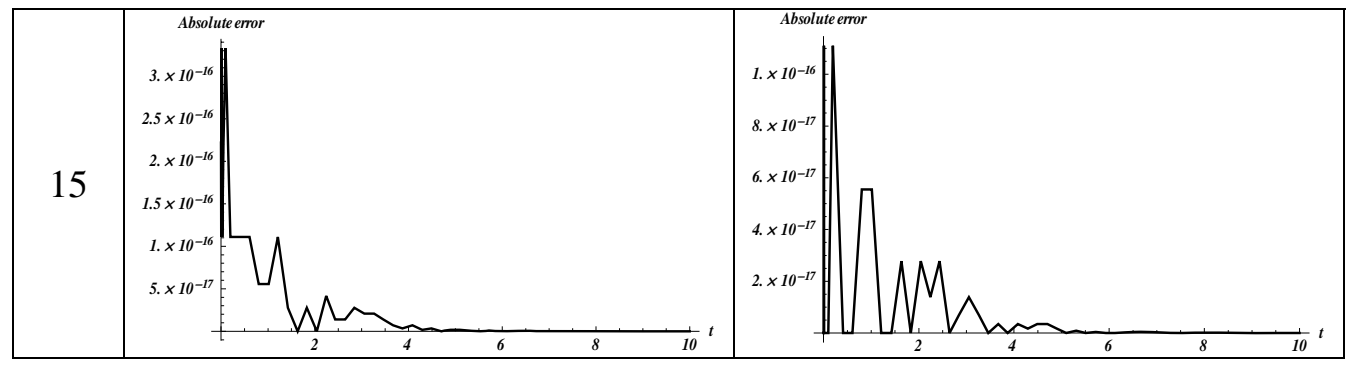

Table 4: Figures of the absolute error between the exact solution of (15) and different forms of the truncated series and Padé approximants using $h=1$.

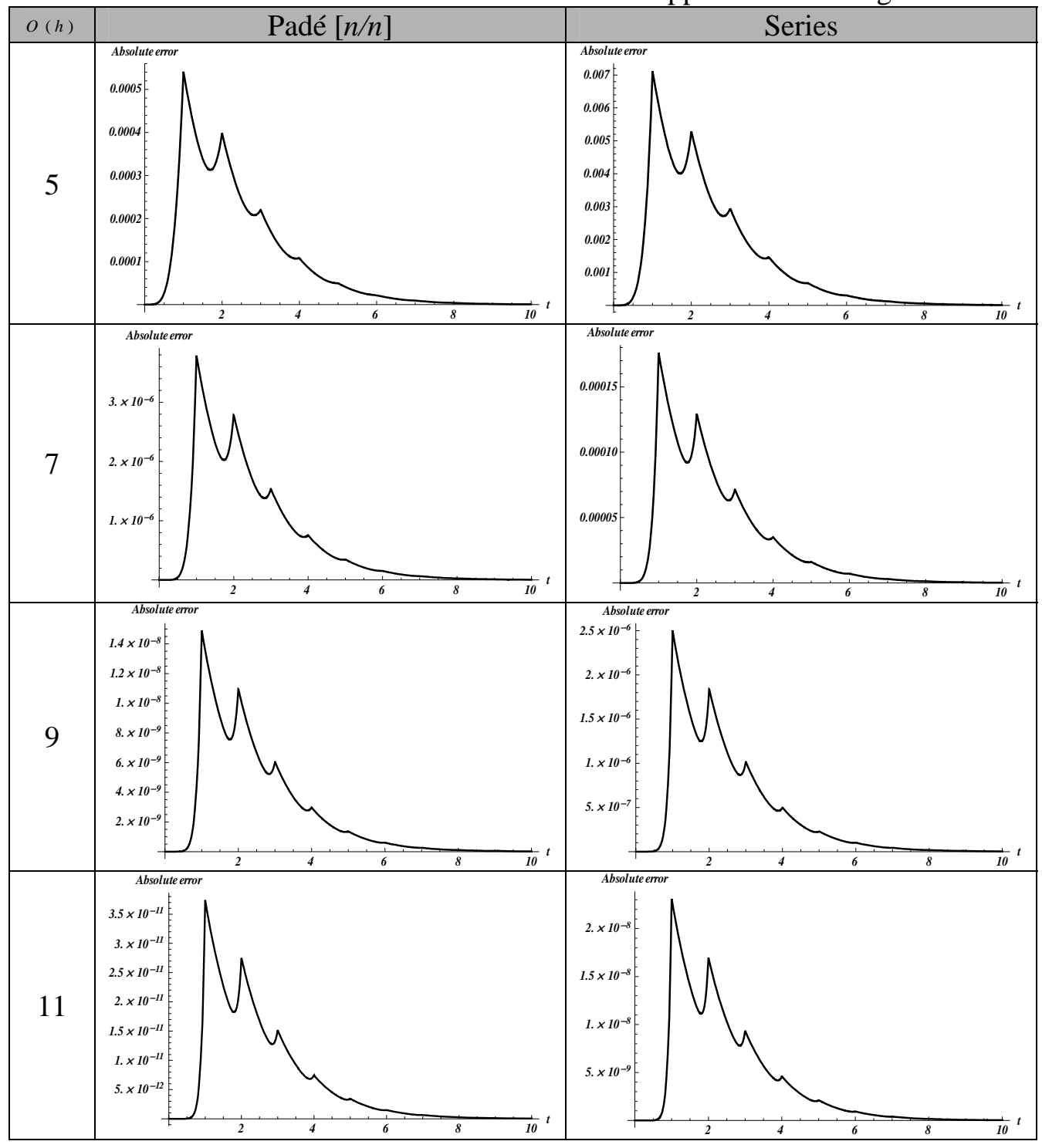




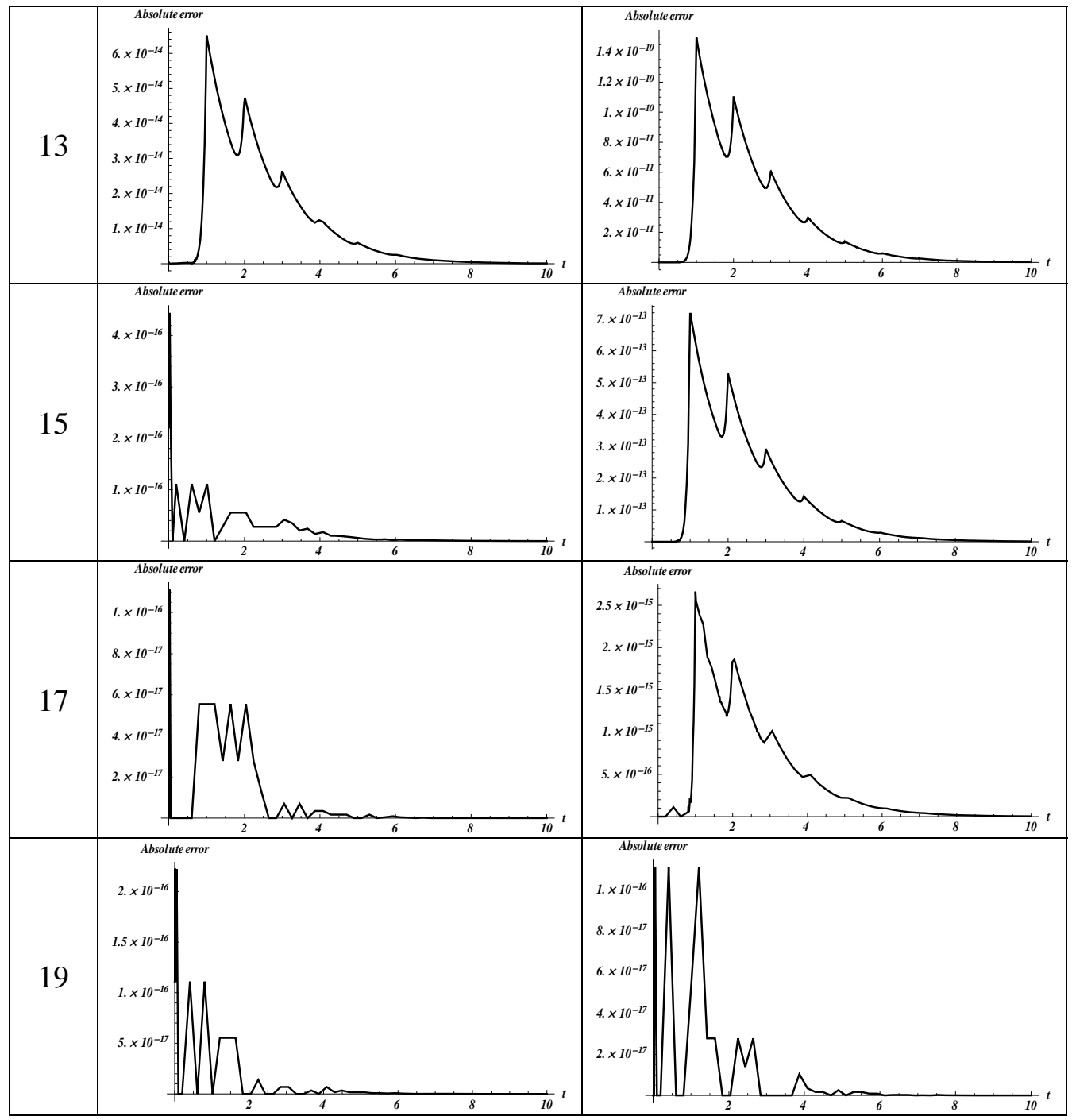

\subsection{Illustrative Example 2:}

Consider the ordinary differential equation

$$
\frac{d u}{d t}=u, \quad u(0)=1
$$

which is a linear problem and has the exact solution

$$
u(t)=e^{t}
$$

Following the same procedures in example 1, we obtain:

$$
\begin{gathered}
c_{0}=f_{m}, \\
c_{1}=\frac{c_{0}}{1}=f_{m},
\end{gathered}
$$


Piecwise Analytic Method

$$
\begin{gathered}
c_{2}=\frac{c_{1}}{2}=\frac{f_{m}}{2}, \\
c_{3}=\frac{c_{2}}{3}=\frac{f_{m}}{3 !}, \\
\vdots \\
c_{n}=\frac{c_{n-1}}{n}=\frac{f_{m}}{n !},
\end{gathered}
$$

Substituting by (27) into (3), we obtain the approximate analytic Taylor series solution

$$
\begin{aligned}
U_{m}(t) \simeq & f_{m}\left(1+\left(t-t_{m}\right)+\frac{\left(t-t_{m}\right)^{2}}{2 !}+\frac{\left(t-t_{m}\right)^{3}}{3 !}+\frac{\left(t-t_{m}\right)^{4}}{4 !}+\frac{\left(t-t_{m}\right)^{5}}{5 !}+\right. \\
& \left.+\frac{\left(t-t_{m}\right)^{6}}{6 !}+\frac{\left(t-t_{m}\right)^{7}}{7 !}+\frac{\left(t-t_{m}\right)^{8}}{8 !}\right), \quad t \in\left[t_{m}, t_{m+1}\right]
\end{aligned}
$$

Substituting by (27) into (14) for obtaining $p^{\prime} s$ and $q$ 's and then substituting by them into (4), we obtain Padé approximants $[4 / 4]$

$$
U_{m}(t) \simeq \frac{f_{m}\left(1680-840\left(t-t_{m}\right)+180\left(t-t_{m}\right)^{2}-20\left(t-t_{m}\right)^{3}+\left(t-t_{m}\right)^{4}\right)}{1680-840\left(t-t_{m}\right)+180\left(t-t_{m}\right)^{2}-20\left(t-t_{m}\right)^{3}+\left(t-t_{m}\right)^{4}}, t \in\left[t_{m}, t_{m+1}\right]
$$

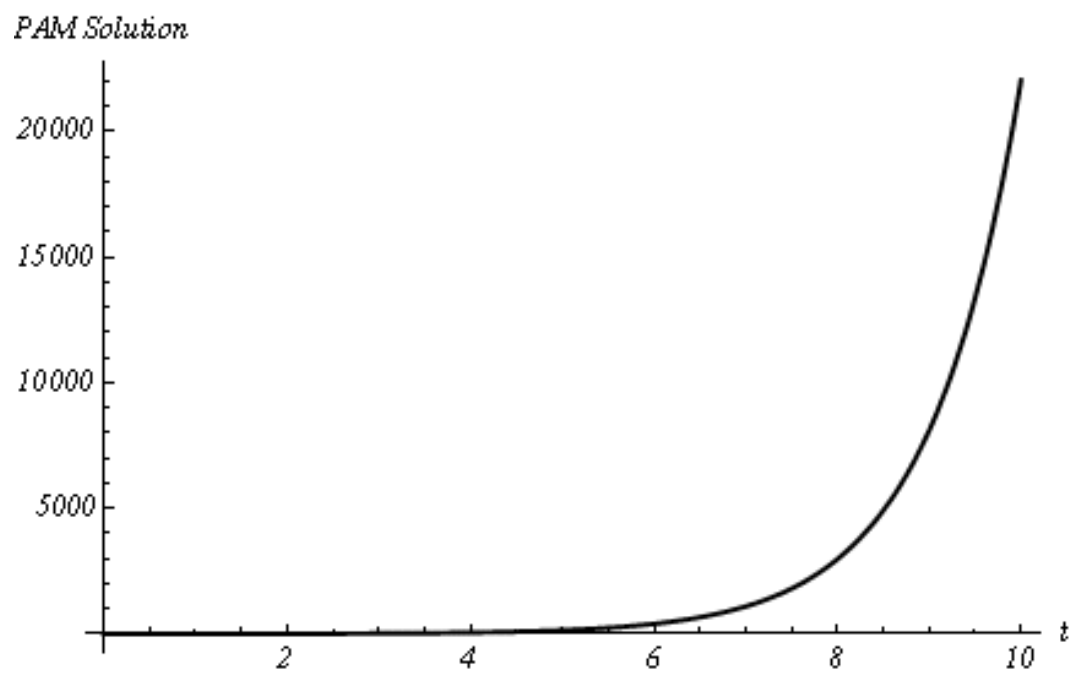

Figure 3: The PAM solution (29) with $h=.01$.

Figure 3 shows the PAM solution (29) using $h=.01$. Table 5 shows the absolute error between the exact solution of the ordinary differential equation (25) (which is unbounded) and truncated series solution (28) and Padé approximants solution $[4 / 4]$ (29), which both have $O\left(h^{9}\right)$, for different values of $h$. Table 6 
and Table 7 show figures of the absolute error between the exact solution of (15) and different forms of the truncated series and Padé approximants using $h=0.01$ and $h=1$ respectively.

Table 5: Figures of the absolute error between the exact solution of the ordinary differential equation (25) and truncated series solution (28) and Padé approximants solution $[4 / 4]$ (29) for different values of $h$.

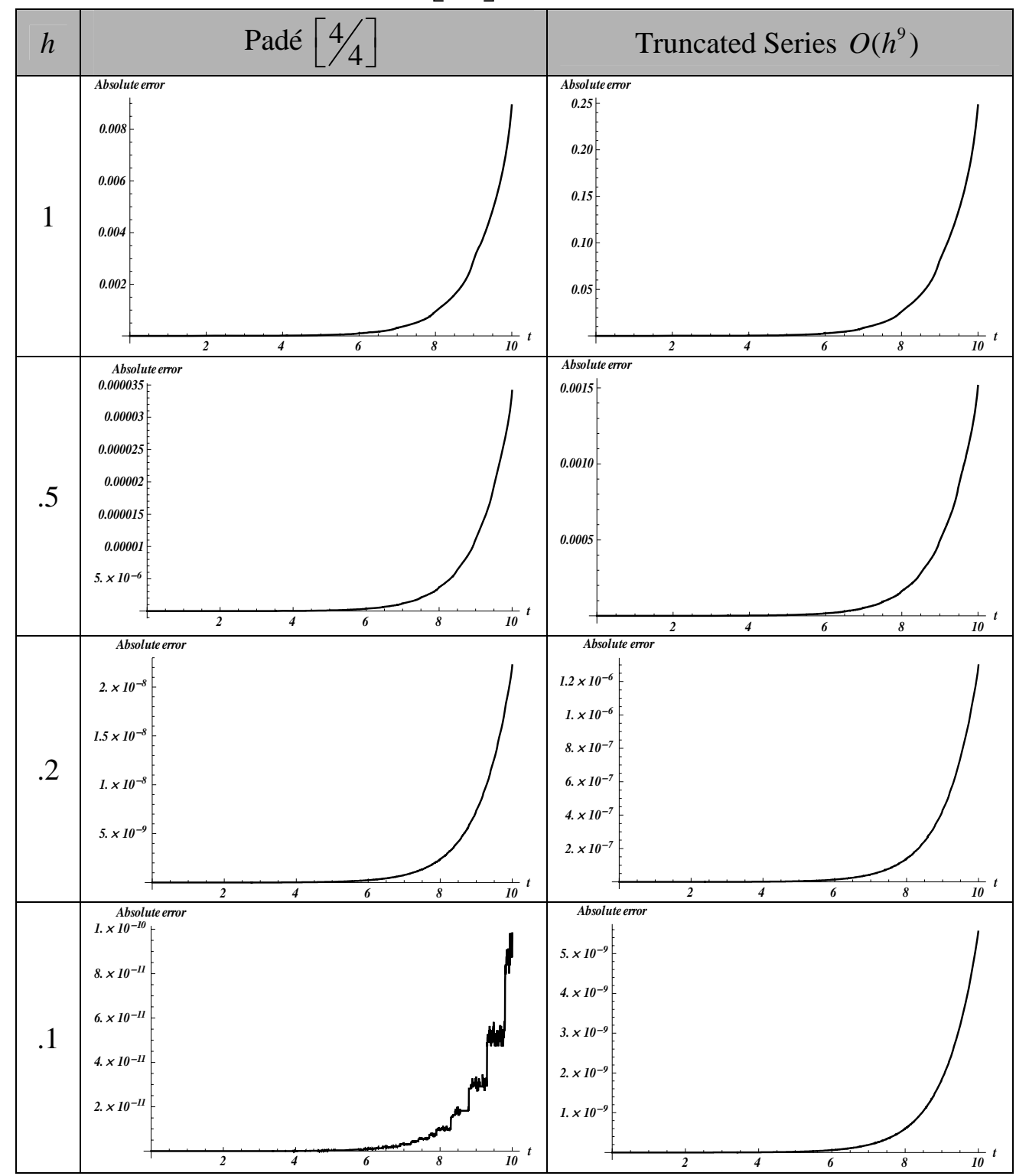




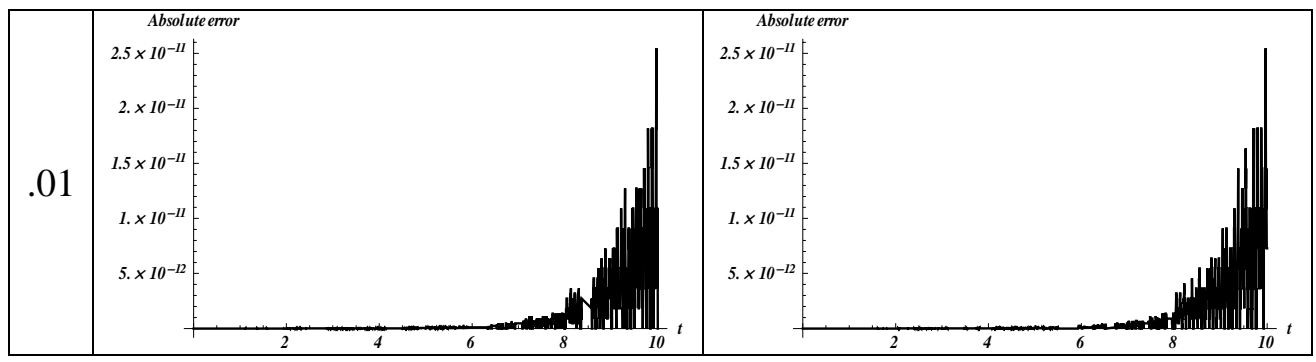

Table 6: Figures of the absolute error between the exact solution of (25) and different forms of the truncated series and Padé approximants $(h=0.01)$.

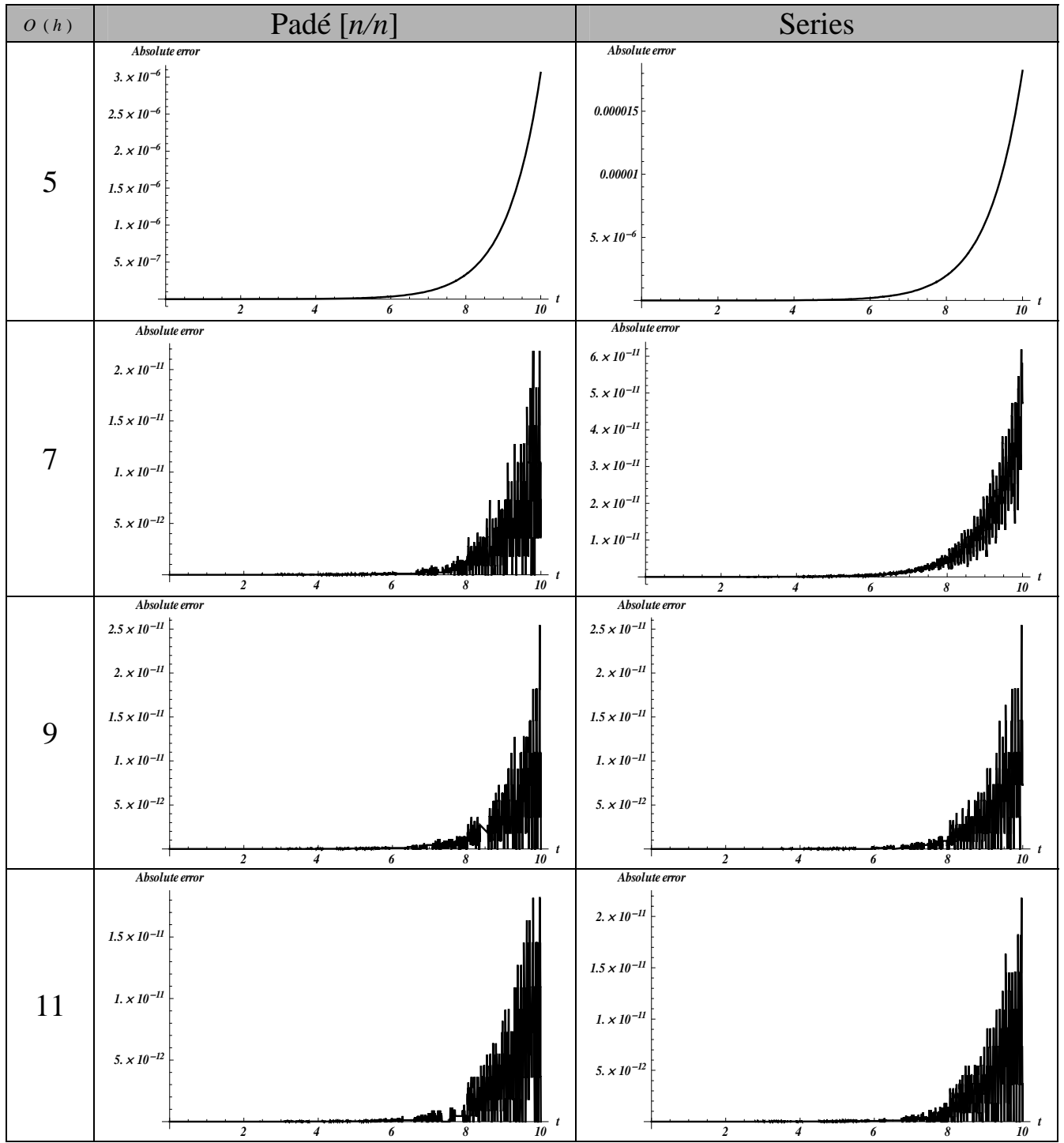




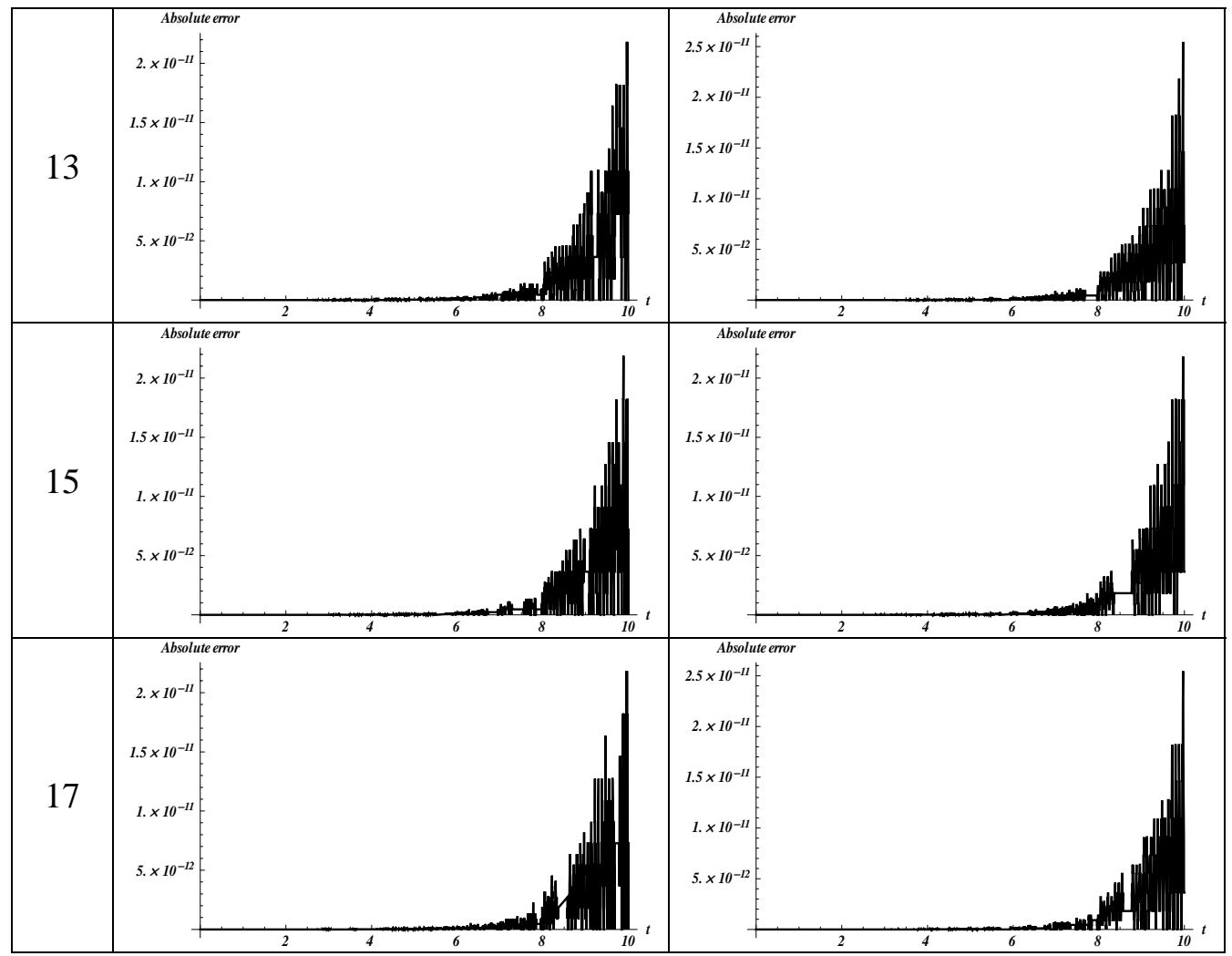

Table 7: Figures of the absolute error between the exact solution of (25) and different forms of the truncated series and Padé approximants fixing the time step $h$ at 1.

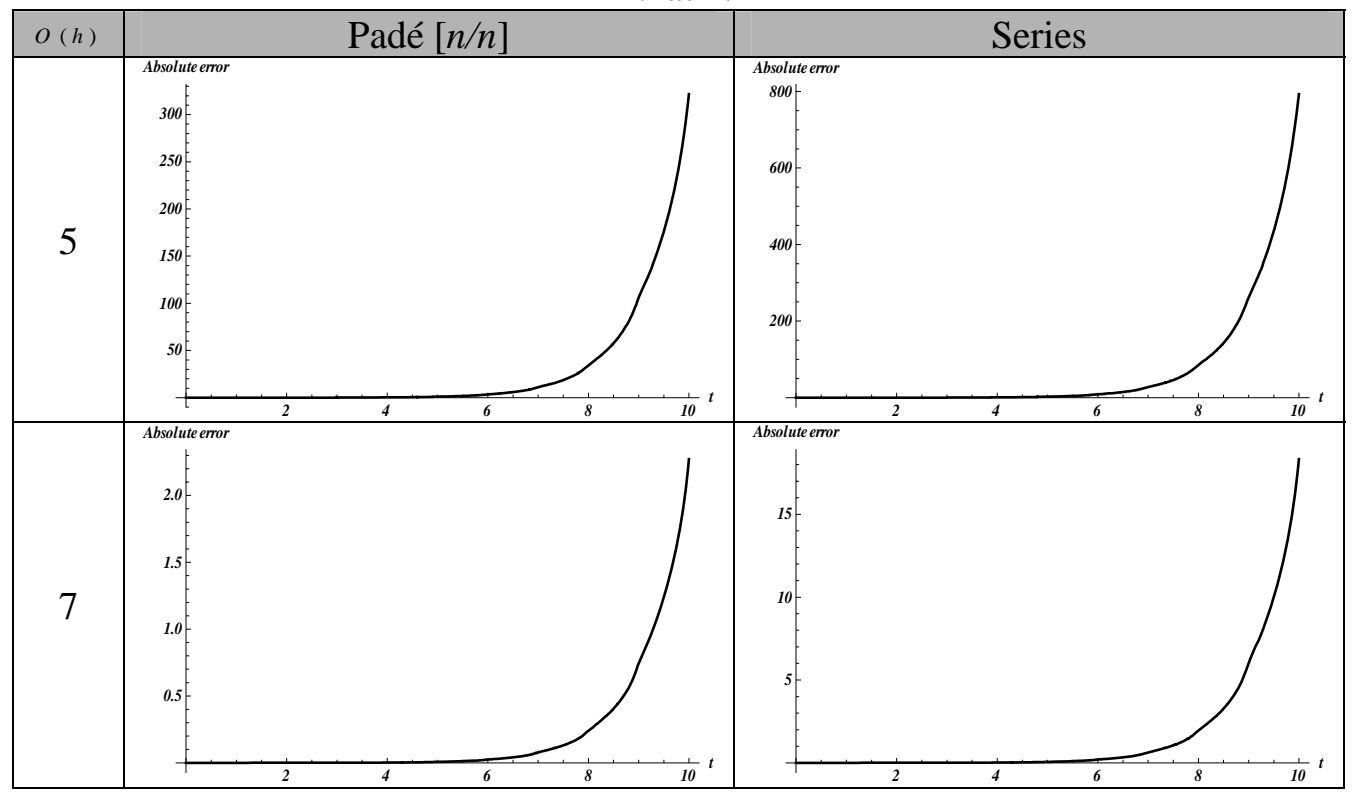


Piecwise Analytic Method

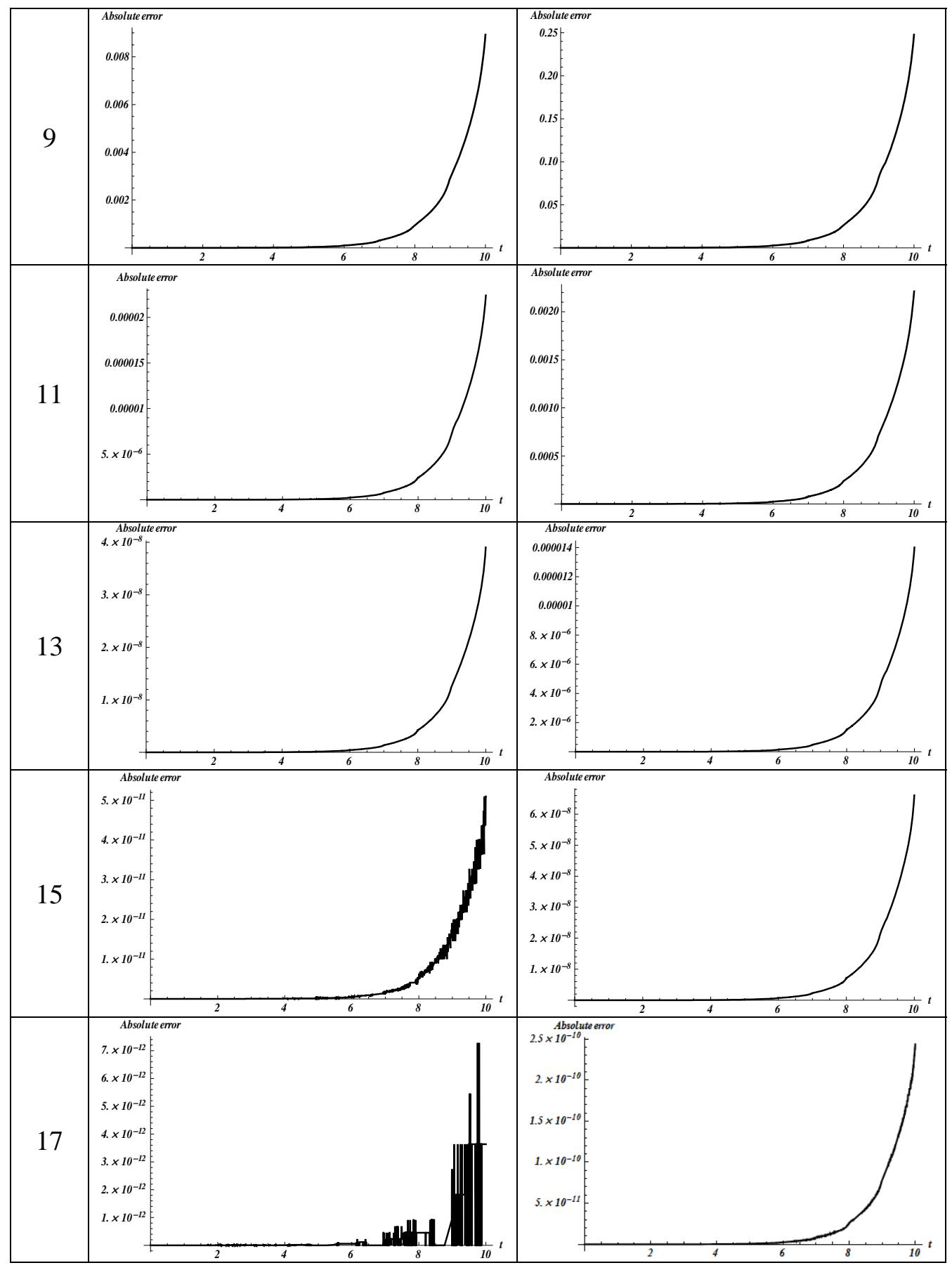




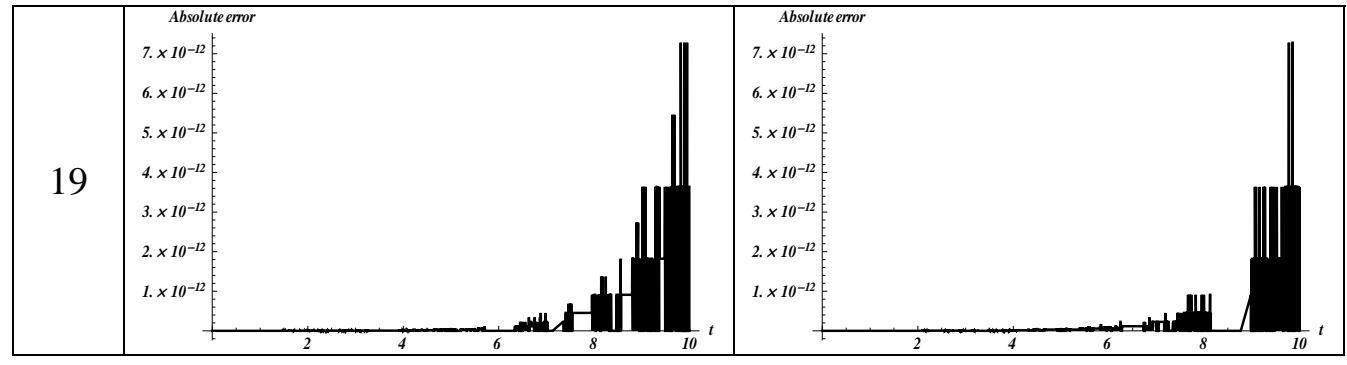

\subsection{Illustrative Example 3: (Non-Linear \& Homogenous)}

Consider the nonlinear homogenous differential equation

$$
u^{\prime}(t)=-u^{2}, \quad u(0)=-1 \text {. }
$$

Equation (30) has the exact solution

$$
u(t)=\frac{1}{t-1} .
$$

This solution has an important characteristic, it is a rational function and has a singular point at $t=1$. This example is very good to study the effect of the pole on the obtain PAM solution.

Fixing $b=2$ and $h=0.1$ which leads to

$$
n=\frac{b-t_{0}}{h}=\frac{2-0}{0.1}=20 \text { subintervals. }
$$

Secondly, from(30), we define the following differential equation for each subinterval $m$

$$
\frac{d U_{m}}{d t}=-U_{m}^{2}, \quad U_{m}\left(t_{m}\right)=f_{m}, \quad t \in\left[t_{m}, t_{m+1}\right] .
$$

$$
\text { where } f_{m}=U_{m-1}\left(t_{m}\right), U_{-1}\left(t_{0}\right)=u(0)=-1, m=0,1,2, \ldots, n-1 \text {. }
$$

Substituting by $U_{m}(t)=\sum_{n=0}^{s} c_{n}\left(t-t_{m}\right)^{n}$ and its derivatives into (33) leads to

$$
\sum_{n=1}^{s} n c_{n}\left(t-t_{m}\right)^{n-1}=-\left(\sum_{n=0}^{s} c_{n}\left(t-t_{m}\right)^{n}\right)^{2}, \quad c_{0}=f_{m}, \quad t \in\left[t_{m}, t_{m+1}\right] .
$$

Solving (34) leads to:

$$
\begin{gathered}
c_{0}=f_{m}, \\
c_{1}=-\left(c_{0}\right)^{2}=-\left(f_{m}\right)^{2}, \\
c_{2}=-c_{0} c_{1}=\left(f_{m}\right)^{3}, \\
c_{3}=-\left(\left(c_{1}\right)^{2}+c_{1} c_{2}\right)^{2}=-\left(f_{m}\right)^{4}, \\
\vdots \\
c_{n}=-\sum_{k=0}^{n-1} c_{n-k-1} c_{k}=(-1)^{n}\left(f_{m}\right)^{n+1},
\end{gathered}
$$


Substituting by (35) into (3), if we need accuracy $O\left(h^{5}\right)$, we can obtain the approximate analytic Taylor series solution

$U_{m}(t) \simeq f_{m}-f_{m}{ }^{2}\left(t-t_{m}\right)+f_{m}{ }^{3}\left(t-t_{m}\right)^{2}-f_{m}{ }^{4}\left(t-t_{m}\right)^{3}+f_{m}{ }^{5}\left(t-t_{m}\right)^{4} \quad t \in\left[t_{m}, t_{m+1}\right]$

Substituting by (35) into (12) for obtaining $p^{\prime} s$ and $q$ 's then substituting into (4), we obtain the Padé approximants

$$
U_{m}(t)=\frac{f_{m}}{1+f_{m}\left(t-t_{m}\right)}, \quad t \in\left[t_{m}, t_{m+1}\right]
$$

if we need accuracy of $O\left(h^{9}\right)$, we can obtain the approximate analytic Taylor series

$$
\begin{aligned}
U_{m}(t) \simeq & f_{m}-f_{m}{ }^{2}\left(t-t_{m}\right)+f_{m}{ }^{3}\left(t-t_{m}\right)^{2}-f_{m}{ }^{4}\left(t-t_{m}\right)^{3}+f_{m}{ }^{5}\left(t-t_{m}\right)^{4}- \\
& f_{m}{ }^{6}\left(t-t_{m}\right)^{5}+{f_{m}}^{7}\left(t-t_{m}\right)^{6}-f_{m}{ }^{8}\left(t-t_{m}\right)^{7}+f_{m}{ }^{9}\left(t-t_{m}\right)^{8} \quad t \in\left[t_{m}, t_{m+1}\right]
\end{aligned}
$$

and the Padé approximants will be

$$
U_{m}(t)=\frac{f_{m}}{1+f_{m}\left(t-t_{m}\right)}, \quad t \in\left[t_{m}, t_{m+1}\right]
$$

which is the same as (37).

The Taylor series solutions (36) and (38) are not accepted near the poles because the error will be very large and not accepted at all. In the other side, observe the Padé approximants solutions (37) and (39), you will find that the exact solution (31) ,(37) and (39) are same. Figure 4 shows the PAM solution using Padé which is the same as the exact solution (31).

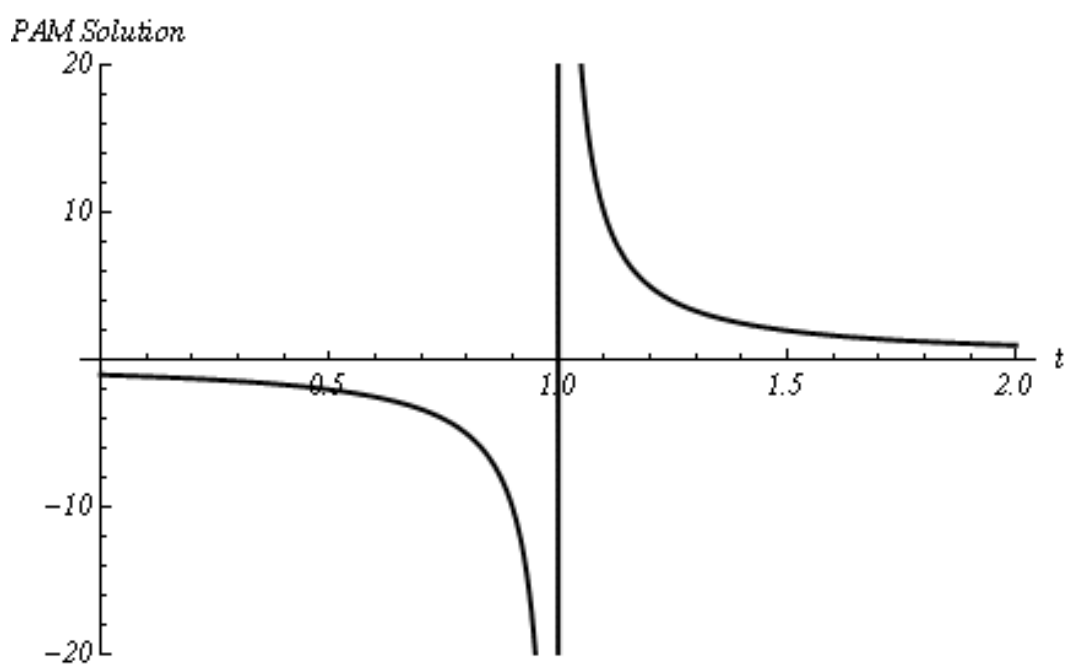

Figure 4: The PAM solution (Padé) of problem (30). 


\subsection{Illustrative Example 4: (Non-Linear Non-Homogenous)}

Consider the differential equation

$$
u^{\prime}(t)=u^{2}+1, \quad u(0)=0 .
$$

This is a nonlinear problem which has the exact solution

$$
u(t)=\tan (t)
$$

This exact solution of equation (40) has an infinite number of singular points at $t=\frac{\pi}{2} \pm(n \pi), n=0,1,2, \ldots$. In this example, we will see the effect of these singular points on the solution.

From (40), we can define the following differential equation for each subinterval $m$

$$
\frac{d U_{m}}{d t}=U_{m}^{2}+1, \quad U_{m}\left(t_{m}\right)=f_{m}, \quad t \in\left[t_{m}, t_{m+1}\right] .
$$

where $f_{m}=U_{m-1}\left(t_{m}\right), U_{-1}\left(t_{0}\right)=u(0)=0, m=0,1,2, \ldots, n-1$

Substituting $U_{m}(t)=\sum_{n=0}^{s} c_{n}\left(t-t_{m}\right)^{n}$ and its derivatives into (42) leads to

$$
\sum_{n=1}^{s} n c_{n}\left(t-t_{m}\right)^{n-1}=\left(\sum_{n=0}^{s} c_{n}\left(t-t_{m}\right)^{n}\right)^{2}+1, \quad c_{0}=f_{m}, \quad t \in\left[t_{m}, t_{m+1}\right] .
$$

Solving (43) leads to:

$$
\begin{gathered}
c_{0}=f_{m}, \\
c_{1}=1+f_{m}{ }^{2}, \\
c_{2}=f_{m}\left(1+f_{m}{ }^{2}\right), \\
c_{3}=\frac{1}{3}\left(1+4 f_{m}{ }^{2}+3 f_{m}{ }^{4}\right), \\
c_{4}=\frac{1}{3} f_{m}\left(2+5 f_{m}{ }^{2}+3 f_{m}{ }^{4}\right), \\
c_{5}=\frac{1}{15}\left(2+17 f_{m}{ }^{2}+30 f_{m}{ }^{4}+15 f_{m}{ }^{6}\right), \\
c_{7}=\frac{1}{45} f_{m}\left(17+77 f_{m}{ }^{2}+105 f_{m}{ }^{4}+45 f_{m}{ }^{6}\right), \\
c_{8}=\frac{1}{315} f_{m}\left(62+440 f_{m}{ }^{2}+1008 f_{m}{ }^{4}+945 f_{m}{ }^{6}+315 f_{m}{ }^{8}\right),
\end{gathered}
$$

Substituting by (44) into(3), we obtain the approximate analytic Taylor series solution. 


$$
\begin{aligned}
& U_{m}(t) \simeq f_{m}+\left(1+f_{m}{ }^{2}\right)\left(t-t_{m}\right)+\left(f_{m}\left(1+f_{m}{ }^{2}\right)\right)\left(t-t_{m}\right)^{2}+ \\
& \left(\frac{1}{3}\left(1+4 f_{m}{ }^{2}+3 f_{m}{ }^{4}\right)\right)\left(t-t_{m}\right)^{3}+\left(\frac{1}{3} f_{m}\left(2+5 f_{m}{ }^{2}+3 f_{m}{ }^{4}\right)\right)\left(t-t_{m}\right)^{4}, \quad t \in\left[t_{m}, t_{m+1}\right]
\end{aligned}
$$

Substituting by (44) into (12) for obtaining $p^{\prime} s$ and $q^{\prime} s$ then substituting by them into(4), we obtain the Padé approximants

$$
U_{m}(t) \simeq \frac{-3 f_{m}-3\left(t-t_{m}\right)+f_{m}\left(t-t_{m}\right)^{2}}{-3+3 f_{m}\left(t-t_{m}\right)+\left(t-t_{m}\right)^{2}}, \quad t \in\left[t_{m}, t_{m+1}\right]
$$

if we need accuracy of $O\left(h^{9}\right)$, we can obtain the approximate analytic Taylor series solution

$$
\begin{aligned}
& \left.U_{m}(t)\right] \simeq f_{m}+\left(t-t_{m}\right)\left(1+f_{m}^{2}\right)+\left(t-t_{m}\right)^{2} f_{m}\left(1+f_{m}^{2}\right)+\frac{1}{3}\left(t-t_{m}\right)^{3}\left(1+4 f_{m}^{2}+3 f_{m}^{4}\right)+ \\
& \quad \frac{1}{3}\left(t-t_{m}\right)^{4} f_{m}\left(2+5 f_{m}^{2}+3 f_{m}^{4}\right)+\frac{1}{15}\left(t-t_{m}\right)^{5}\left(2+17 f_{m}^{2}+30 f_{m}^{4}+15 f_{m}^{6}\right)+ \\
& \quad \frac{1}{45}\left(t-t_{m}\right)^{6} f_{m}\left(17+77 f_{m}^{2}+105 f_{m}^{4}+45 f_{m}^{6}\right)+ \\
& \quad \frac{1}{315}\left(t-t_{m}\right)^{7}\left(17+248 f_{m}^{2}+756 f_{m}^{4}+840 f_{m}^{6}+315 f_{m}^{8}\right)+ \\
& \frac{1}{315}\left(t-t_{m}\right)^{8} f_{m}\left(62+440 f_{m}^{2}+1008 f_{m}^{4}+945 f_{m}^{6}+315 f_{m}^{8}\right), \quad t \in\left[t_{m}, t_{m}+1\right]
\end{aligned}
$$

and Padé approximants

$U_{m}(t) \simeq \frac{105 f_{m}+105\left(t-t_{m}\right)-45 f_{m}\left(t-t_{m}\right)^{2}-10\left(t-t_{m}\right)^{3}+f_{m}\left(t-t_{m}\right)^{4}}{105-105 f_{m}\left(t-t_{m}\right)-45\left(t-t_{m}\right)^{2}+10 f_{m}\left(t-t_{m}\right)^{3}+\left(t-t_{m}\right)^{4}}, t \in\left[t_{m}, t_{m+1}\right]$

The truncated series solution is not suitable for this problem because it has an infinite number of singular points. Figure 5 shows the Padé approximants solution (48) which is obtained by PAM and Figure 6 shows the absolute error between the exact solution (41) and the Padé approximants solution(48). Table 8 shows the absolute error between the exact solution (41) and the Padé approximants solution(48) for different values of $h, t_{0}=0$ and $\frac{\pi}{2}<t<3 \frac{\pi}{2}$. Table 9 shows the absolute error between the exact solution (41) and different forms of Padé approximants solution fixing $h=0.1, t_{0}=0$ and $\frac{\pi}{2}<t<3 \frac{\pi}{2}$. 


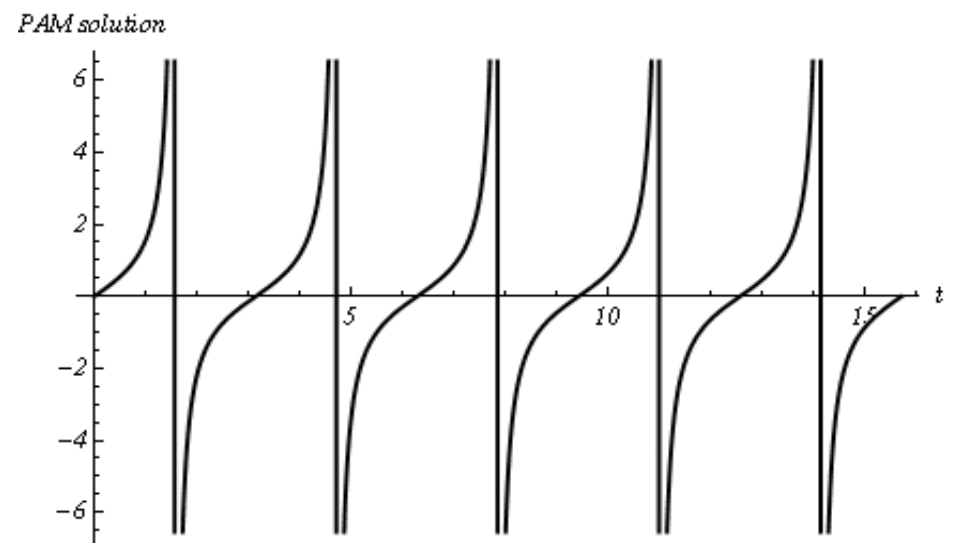

Figure 5: The PAM solution (48) using $h=.01$.

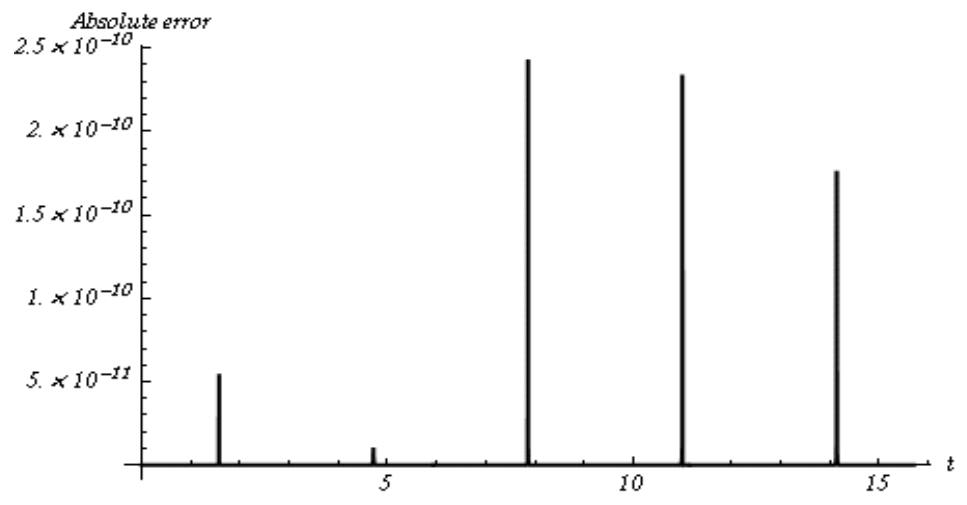

Figure 6: The absolute error between the exact solution (41) and Padé approximants solution(48) using $0<t<5 \pi$ and $h=0.01$.

Table 8: The absolute error between the exact solution (41) and the Padé approximants [4/4](48) for different values of $h, \frac{\pi}{2}<t<3 \frac{\pi}{2}$ and $t_{0}=0$.

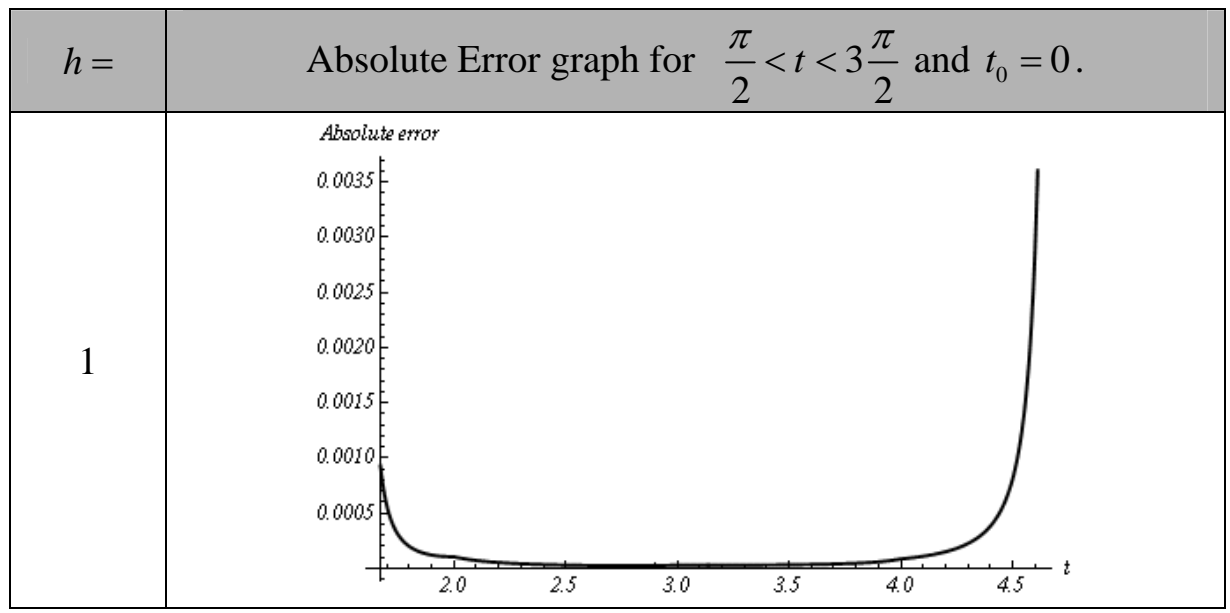




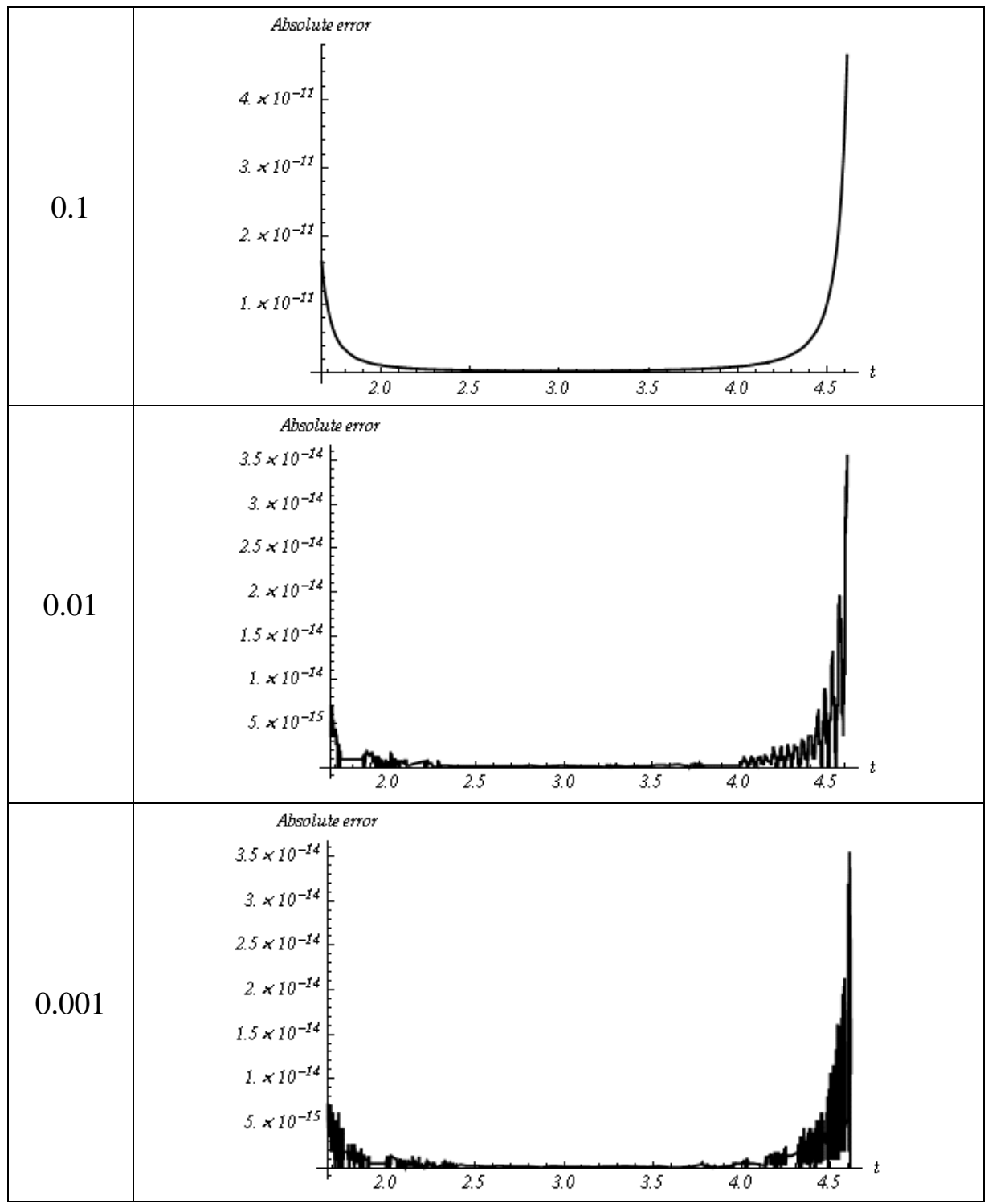


Table 9: The absolute error between the exact solution (41) and different forms of Padé approximants for $h=0.1, \frac{\pi}{2}<t<3 \frac{\pi}{2}$ and $t_{0}=0$.

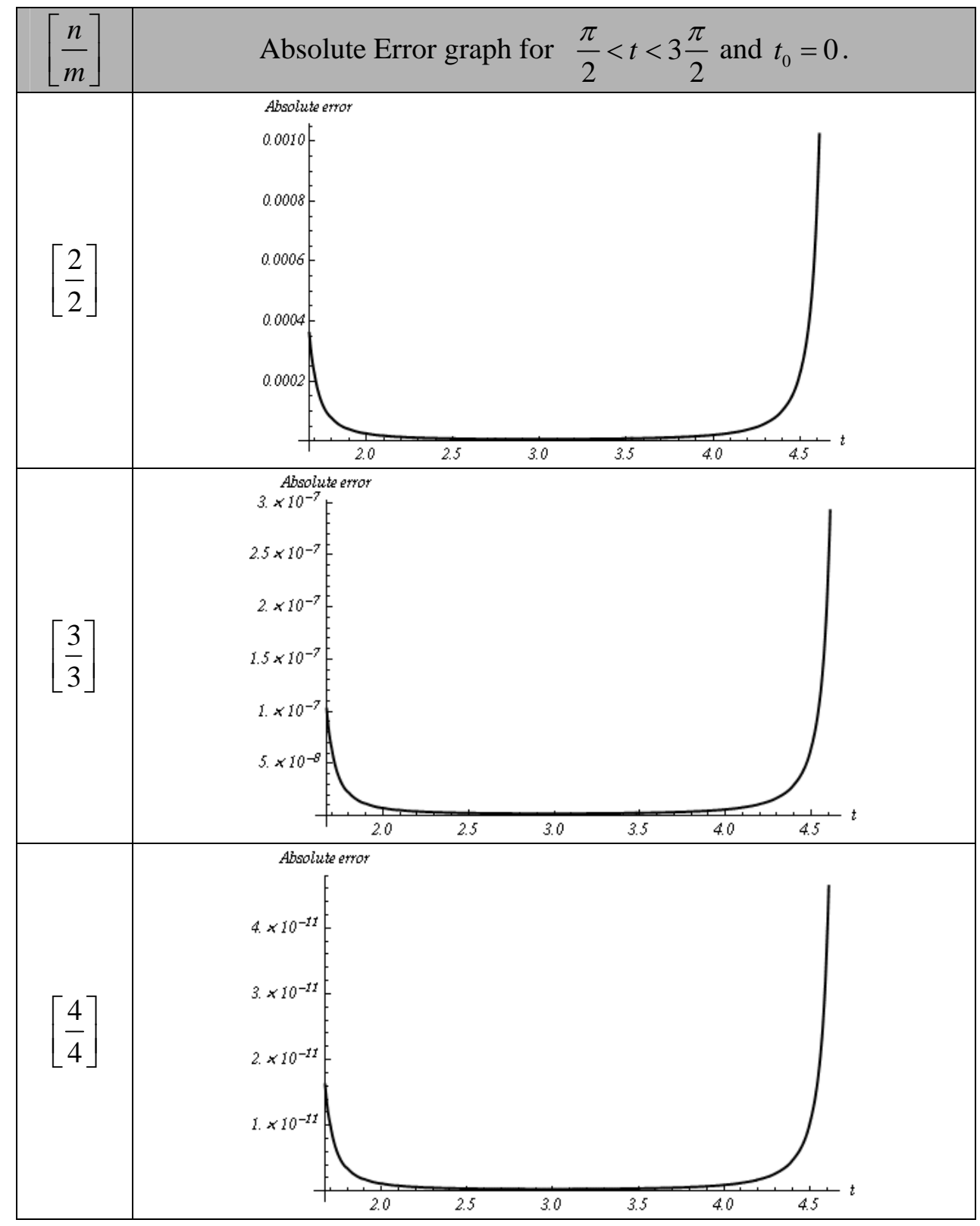




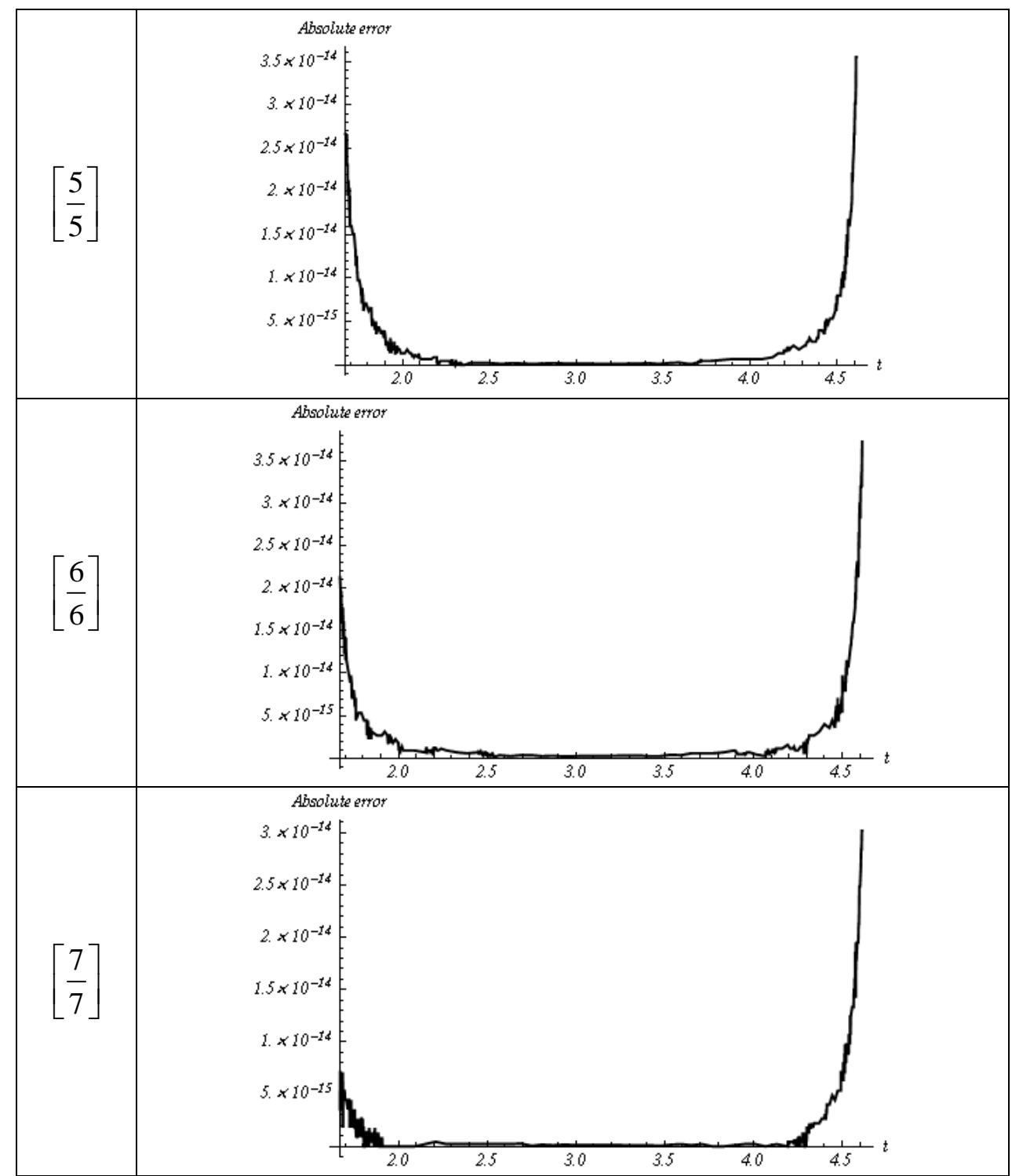

\section{Observation:}

We can observe from the previous illustrative examples 1-4 and the results in tables 2-9 that the absolute error is taking the same shape approximately in each column until specified $h$ or order and then the error is fixed. This isn't logic because the error must not be fixed as $h$ or the order is reduced. The error must approaches to zero as $h$ approaches zero or the order increases. This is because of the roundoff error, the limited specification of the computer device and the mathematical program which I have used. 


\section{Conclusion:}

The piecewise analytic method is an efficient method for solving any ordinary differential equation. We can control the accuracy of the obtained solution as needed. We use the Padé approximants when the solution contains poles or singular points and it is suitable for large interval length. The truncated series solution is faster than Padé approximants but the Padé is more accurate than truncated series.

\section{References:}

1. Arfken, G., "Taylor's Expansion." \$5.6 in Mathematical Methods for Physicists, 3rd ed. Orlando, FL: Academic Press, 1985: p. 303-313.

2. Abramowitz, M. and I.A. Stegun, Handbook of Mathematical Functions with Formulas, Graphs, and Mathematical Tables, 9th printing. New York: Dover, 1972: p. 880.

3. Baker, G.A.J., "The Theory and Application of The Padé Approximant Method." In Advances in Theoretical Physics, Vol. 1 (Ed. K. A. Brueckner). New York: Academic Press, 1965: p. 1-58.

4. Baker, G.A.J., Essentials of Padé Approximants in Theoretical Physics. New York: Academic Press, 1975: p. 27-38.

5. Baker, G.A.J. and P. Graves-Morris, Padé Approximants. New York: Cambridge University Press, 1996.

6. $\quad$ Euler, L., Inst. Calc. Int., 1768. 1: p. 493.

7. Euler, L. and Opera Omnia, Series Prima, Vol. 11, Leipzig and Berlin, 1913.

8. Euler, L. and Opera Omnia, Series Prima, Vol. 12, Leipzig and Berlin, 1914.

9. Butcher, J.C., Numerical Analysis of Ordinary Differential Equations: Runge-Kutta and Linear Methods. Wiley,New York, 1987.

10. Kutta, W., Beitrag zur näherungweisen Integration totaler Differentialgleichungen ZAMP, 1901. 46: p. 435.

11. Runge, C., Uber die numerische Auflosung von Differentialgleichungen. Math. Ann., 1895. 46: p. 167.

12. Adomian, G., Solving Frontier Problem of Physics: the Decomposition Method. MA: Kluwer Academic Publishers, Boston, 1994.

13. Tamer A, A., Improved Adomian decomposition method (solving nonlinear non-homogenous initial value problem). Journal of the Franklin Institute, 2011. 348(6): p. 1035-1051.

14. Abassy, T.A., Improved Adomian decomposition method. Computers \& Mathematics with Applications, 2010. 59(1): p. 42-54.

15. Tamer A, A., Modified variational iteration method (non-homogeneous initial value problem). Mathematical and Computer Modelling, 2012. 55(3â€“4): p. 1222-1232. 
16. Abassy, T.A., Modified variational iteration method (nonlinear homogeneous initial value problem). Computers \& Mathematics with Applications, 2010. 59(2): p. 912-918.

17. He, J.-H., Homotopy perturbation technique. Computer Methods in Applied Mechanics and Engineering, 1999(178): p. 257-262.

18. SJ., L., The proposed homotopy analysis technique for the solution of nonlinear problems. PhD thesis. Shanghai: Shanghai Jiao Tong University, 1992. 